\title{
LOCAL REGULARIZATION FOR THE NONLINEAR INVERSE AUTOCONVOLUTION PROBLEM*
}

\author{
ZHEWEI DAI ${ }^{\dagger}$ AND PATRICIA K. LAMM $\ddagger$
}

\begin{abstract}
We develop a local regularization theory for the nonlinear inverse autoconvolution problem. Unlike classical regularization techniques such as Tikhonov regularization, this theory provides regularization methods that preserve the causal nature of the autoconvolution problem, allowing for fast sequential numerical solution $\left(\mathcal{O}\left(r N^{2}-r^{2} N\right)\right.$ flops where $r \ll N$ for the method discussed in this paper as applied to the nonlinear problem; in comparison, the cost for Tikhonov regularization applied to a general linear problem is $\mathcal{O}\left(N^{3}\right)$ flops). We prove the convergence of the regularized solutions to the true solution as the noise level in the data shrinks to zero and supply convergence rates for the case of both $L_{2}$ and continuous data. We propose several regularization methods and provide a theoretical basis for their convergence; of note is that this class of methods does not require an initial guess of the unknown solution. Our numerical results confirm effectiveness of the methods, with results comparing favorably to numerical examples found in the literature for the autoconvolution problem (e.g., [13] for examples using Tikhonov regularization with total variation constraints, and [16] for examples using the method of Lavrent'ev); this especially seems to be true when it comes to the recovery of sharp features in the unknown solution. We also show the effectiveness of our method in cases not covered by the theory.
\end{abstract}

Key words. local regularization of inverse problems, nonlinear Volterra problem, numerical methods, inverse autoconvolution problem

AMS subject classifications. 65R20, 65R30, 65R32, 45Q05

1. Introduction. In this paper we develop a local regularization theory for the inverse autoconvolution problem of finding $\bar{x} \in L_{2}(0, T)$ solving

$$
G(x)=f,
$$

where $G$ is the nonlinear Volterra operator given by

$$
G(x)(t)=\int_{0}^{t} x(t-s) x(s) d s, \quad \text { a.e. } \mathrm{t} \in(0, \mathrm{~T})
$$

and where $f \in \operatorname{Range}(G) \subseteq L_{2}(0, T)$. Without loss of generality we will henceforth let $T=1$.

Autoconvolution has been of interest to scientists for decades because of its applications in various fields. It arises in stochastics where the density function of a continuous random variable $V$ is reconstructed after observing the density function of the random variable $S=V_{1}+V_{2}$, where $V_{1}$ and $V_{2}$ are identically and independently distributed random variables of $V$ [14]. Another application of autoconvolution occurs in spectroscopy. Baumeister presents in [1] a reference list of physically motivated papers concerning this class of problems. He also discusses in detail the mathematical model of deconvolution of "appearance potential"(AP) spectra to investigate electronic properties of solids in their surface region. In this context, the density of

*This work was supported in part by the National Science Foundation under contract numbers NSF DMS-0104003 and DMS-0405978. Parts of the research was completed while both authors were at the Institute for Pure and Applied Mathematics (IPAM) at UCLA. We wish to thank IPAM for providing the financial support and hospitable environment conducive to productive research.

$\dagger$ Department of Mathematics, Alma College, Alma, MI 48801 dai@alma.edu

$\ddagger$ Department of Mathematics, Michigan State University, East Lansing, MI 48824-1027 lamm@math.msu.edu 
unoccupied states in the surface region of a solid is recovered from the measured AP-spectrum data.

Properties of the autoconvolution problem have been explored extensively in [11, $12,13,14]$ (to name just a few of the references in this area) and include analysis of ill-posedness of the problem due to lack of continuous dependence of solutions $\bar{x}$ on data $f$. Indeed, for $D(G) \equiv\left\{x \in L_{2}(0,1), x(t) \geq 0\right.$, a.e. $\left.t \in(0,1)\right\}$, the operator $G: D(G) \subset L_{2}(0,1) \mapsto L_{2}(0,1)$ is such that the inverse autoconvolution operator $G^{-1}$ is discontinuous at every point $f$ in the range of $G$; i.e., the autoconvolution equation is locally ill-posed at every point $x$ in $D(G)$. Degrees of ill-posedness for the autoconvolution equation are discussed in [12] and [14]. In short, we expect a correlation between the degree of ill-posedness and both the smoothness of the solution $\bar{x}$ and the behavior of $\bar{x}$ at 0 .

Various regularization methods have been studied for the autoconvolution equation. One can utilize Tikhonov regularization theory for nonlinear inverse problems since the autoconvolution operator $G$ is continuous and weakly closed on $D(G)$ and $G$ has a compact Fréchet derivative at all $x \in L_{2}(0,1)$ satisfying assumptions needed to guarantee stability in the nonlinear Tikhonov theory $[9,14,30]$. However, as is well-known in the case of the linear Volterra problem, classical regularization methods such as Tikhonov regularization have inherent disadvantages in solving Volterra problems; indeed, the use of Tikhonov regularization transforms problems that are structurally causal in nature into non-causal or full-domain problems (see, e.g., [24] for a discussion of these difficulties). Causal-based methods have advantages because they are sequential in nature, and we'll see in Section 5.1 that the cost of the local regularization algorithm discussed in that section is $\mathcal{O}\left(r N^{2}-r^{2} N\right)$ where $r \ll N$, while the cost for Tikhonov regularization on a general linear problem is $\mathcal{O}\left(N^{3}\right)$.

In addition, source conditions needed for convergence rates for Tikhonov regularization as applied to the nonlinear problem (1.1) require that $\bar{x}-x^{\star}=G^{\prime}(\bar{x})^{\star} w$ for some $w \in L_{2}(0,1)[10]$, where $x^{\star}$ is an initial guess for $\bar{x}$ and $\bar{x}$ is the true solution of (1.1). It is not hard to show that the source condition requires that $x^{\star}(1)=\bar{x}(1)$; since $x^{\star}$ is part of the Tikhonov algorithm, the method then requires knowledge of the value of the unknown solution $\bar{x}$ at $t=1$.

In the late 1960's, J. V. Beck developed a regularization scheme for the discretized inverse heat conduction problem (IHCP), a linear Volterra inverse problem, which retains the causal nature of the original problem [2]. Because of its sequential formulation, the numerical implementation of the Beck method is also more efficient than classical regularization methods. In the mid-1990's, the second author established the theoretical basis for the convergence of the sequential local regularization method, and Beck's approach was generalized to a wide class of linear first-kind Volterra problems $[17,18,19]$. While Beck's method was an approach developed to handle a finite dimensional approximation of the IHCP, the current theory of local regularization methods can be placed in both finite and infinite dimensional settings. In addition, the theory has been extended more recently to nonlinear Volterra problems of Hammerstein type,

$$
\int_{0}^{t} k(t-s) g(x(s), s) d s=f(t), \quad \text { a.e. } t \in(0, T) .
$$

In [23], the special structure of this equation was coupled with linear convergence theory so that local regularization was used to solve

$$
\int_{0}^{t} k(t-s) v(s) d s=f(t) \quad \text { a.e. } t \in(0, T),
$$


$v$, and then $u$ was recovered as a solution of the nonlinear problem $v(t)=g(u(t), t)$ for all $t \in[0,1]$. More recently in [26] this work has been modified so that the nonlinear equation (1.3) need only be solved on an initial (small) interval $[0, \varepsilon]$; then for $t>\varepsilon$, the solution $x$ is stably recovered explicitly and sequentially from a local regularization equation which is an extension of the method found in [23].

In [16], Janno applied the Lavrent'ev regularization method to the autoconvolution equation and provided both theoretical convergence estimates and numerical examples. The advantage of Lavrent'ev method is that it preserves the causal nature of Volterra problems and therefore leads to a fast sequential method. The drawback for the method is that it requires and depends on an initial guess $x^{\star}$ of the true solution $\bar{x}$, and it must be that $x^{\star}(0)=\bar{x}(0)$ which means that $\bar{x}(0)$ must be known in advance (or at least computed using regularized differentiation of noisy data; we note that in Section 5 we propose an algorithm which approximates $\bar{x}(0)$ in exactly the same way). Performance of both the Lavrent'ev and Tikhonov methods seem fairly dependent on the choice of the initial guess $x^{\star}$ and of its closeness to $\bar{x}$.

In this paper, we develop a local regularization theory for the nonlinear autoconvolution problem of finding $x \in D(G) \subset L_{2}(0,1)$ solving equation (1.1) with $G$ given by (1.2). The result is an effective regularization method which essentially preserves the casual nature of the problem and does not require an initial guess of the true solution (such as is needed for the Lavrent'ev method and Tikhonov method).

2. The Regularized Autoconvolution Equation. We will first motivate the equation which will become the basis for our regularized approximation of the true solution $\bar{x}$ of (1.1). We assume that our data and solution are available on an extended interval $[0,1+\bar{R}]$ for some small $\bar{R} \in(0,1]$ and note that this can always be accomplished by simply decreasing the size of our original interval slightly. Thus, for any $0<R<\bar{R}, \bar{x}$ solves

$$
\int_{0}^{t+\rho} x(t+\rho-s) x(s) d s=f(t+\rho), \quad \text { a.e. } t \in(0,1), \rho \in(0, R) .
$$

We split the integral at $\rho$ and $t$, then change the variable of integration to obtain

$$
2 \int_{0}^{\rho} x(t+\rho-s) x(s) d s+\int_{\rho}^{t} x(t+\rho-s) x(s) d s=f(t+\rho)
$$

for a.e. $t \in(0,1), \rho \in(0, R)$. In order to consolidate the local future information introduced by the variable $\rho$, we follow the idea of local regularization in the case of the linear Volterra problem (see, e.g., $[17,18])$ and integrate both sides of the equation (2.1) with respect to a suitable measure $\eta=\eta(\rho)>0$ (which we will clarify later) on $[0, R]$, leading to the equation

$$
\begin{aligned}
2 \int_{0}^{R} \int_{0}^{\rho} x(t+\rho-s) x(s) d s d \eta(\rho)+ & \int_{0}^{R} \int_{\rho}^{t} x(t+\rho-s) x(s) d s d \eta(\rho) \\
& =\int_{0}^{R} f(t+\rho) d \eta(\rho), \quad \text { a.e. } t \in(0,1) .
\end{aligned}
$$

Note that $\bar{x}$ still satisfies equation (2.2) exactly. 
In reality, instead of having the exact data $f$, we only have access to some approximation $f^{\delta}$ to $f$. Because of the local ill-posedness of the original problem (1.1), some form of regularization is needed when the perturbed data $f^{\delta}$ is used in place of the "ideal" data $f$. The results of regularization differ depending on the smoothness of the perturbed data, so we will investigate the properties of our method using data $f^{\delta}$ in two different spaces $\mathcal{F}$ of functions defined on $[0,1+\bar{R}]$. Our assumption regarding data will be as follows:

DEFINITION 2.1. We will say the data $f^{\delta}$ satisfies the $\mathcal{F}$-data assumption if $f^{\delta} \in \mathcal{F}$ and

$$
\left\|f^{\delta}-f\right\|_{\mathcal{F}} \leq \delta
$$

for some $\delta>0$, where either $\mathcal{F}=C[0,1+\bar{R}]$ or $\mathcal{F}=L_{2}(0,1+\bar{R})$.

Returning then to local regularization in the presence of perturbed data $f^{\delta}$, the method is suggested by the idea of momentarily holding $x$ constant on a small local interval $[t, t+R]$, i.e., we replace $x(t+\rho-s)$ by $x(t)$ in the first term of equation (2.2) for values of $\rho, s$ such that $\rho-s \in[0, R]$. Here, the length of this local interval $R$ serves as the regularization parameter. We then obtain the regularization equation

$$
\alpha_{R}(x) x+F_{R}(x)=f_{R}^{\delta}
$$

where for a.e. $t \in(0,1)$,

$$
\begin{aligned}
\alpha_{R}(x) & \equiv 2 \int_{0}^{R} \int_{0}^{\rho} x(s) d s d \eta(\rho) \\
F_{R}(x)(t) & \equiv \int_{0}^{R} \int_{\rho}^{t} x(t+\rho-s) x(s) d s d \eta(\rho), \\
f_{R}^{\delta}(t) & \equiv \int_{0}^{R} f^{\delta}(t+\rho) d \eta(\rho) .
\end{aligned}
$$

The $R$-dependent measure $\eta$ is defined via

$$
\int_{0}^{R} g(\rho) d \eta(\rho) \equiv \int_{0}^{R} g(\rho) \omega(\rho, R) d \rho, \quad g \in L_{2}(0, R),
$$

where the family $\left\{\omega(\cdot, R) \in L_{\infty}(0, R)\right\}_{R \in(0, \bar{R}]}$ is assumed to be such that there exists $\underline{\omega}, \bar{\omega}>0$ independent of $R$ so that

$$
0<\underline{\omega} \leq \omega(\rho, R) \leq \bar{\omega}, \quad \text { a.e. } \rho \in(0, R],
$$

for all $R$ sufficiently small. It follows then that for any real $m \geq 0$ there exist constants $K(m)>0$ independent of $R$ so that

$$
\frac{\int_{0}^{R} \rho^{m} d \eta(\rho)}{\int_{0}^{R} \rho d \eta(\rho)} \leq K(m) R^{m-1}
$$

for all $R>0$ sufficiently small. We note that this class of measures is a special case of the measures discussed in previous papers on local regularization for linear Volterra problems (see, e.g., [17, 18, 19, 25, 27, 28, 31]), and that the discrete measures 
discussed in these references are not suitable for use in (2.7) in the case of $f^{\delta} \in$ $L_{2}(0,1+R)$.

Our main results involving the well-posedness of equation (2.4) and the convergence of the solution $x$ of (2.4) to the true solution $\bar{x}$ of the original autoconvolution equation will be given in the next section. First we will introduce some additional spaces and norms which will facilitate further analysis below of the operator $F_{R}$, as well as the theory given in Section 3.

We will define a new $R$-dependent topology on $L_{2}(0,1)$ via

$$
\langle x, y\rangle_{\sigma, R} \equiv \frac{\mathcal{C}^{2}-1}{R} \int_{0}^{R} e^{-2 \sigma t} x(t) y(t) d t+\int_{0}^{1} e^{-2 \sigma t} x(t) y(t) d t,
$$

where $\sigma>0$ and $\mathcal{C}>1$, with further conditions on these constants to be specified later. We will designate by $\|\cdot\|_{\sigma, R}$ the usual norm associated with this inner product, and let $L_{2}^{\sigma, R}(0,1)$ denote the space $L_{2}(0,1)$ coupled with this $R$-dependent topology. The closed ball in $L_{2}^{\sigma, R}(0,1)$ centered at $x_{0}$ with radius $r$ will be defined by

$$
\mathcal{B}\left(x_{0}, r\right)=\left\{z \in L_{2}^{\sigma, R}(0,1),\left\|z-x_{0}\right\|_{\sigma, R} \leq r\right\} .
$$

The following lemma provides a technical estimate useful in the theory which follows.

Lemma 2.2. Let $g \in L_{\infty}(0,1)$. Then for $\sigma, \mathcal{C}$ defined above and $R \in(0, \bar{R}]$,

$$
\|g\|_{\sigma, R} \leq \mathcal{C}\|g\|_{\infty}
$$

Proof. For any $T \in(0,1], \int_{0}^{T} e^{-2 \sigma t}|g(t)|^{2} d t \leq T\|g\|_{\infty}^{2}$ so that

$$
\|g\|_{\sigma, R}^{2} \leq\left(\frac{\mathcal{C}^{2}-1}{R} R+1\right)\|g\|_{\infty}^{2}
$$

Lemma 2.3. The operator $F_{R}: L_{2}^{\sigma, R}(0,1) \rightarrow L_{2}^{\sigma, R}(0,1)$ as defined in (2.6) is Fréchet differentiable with

$$
F_{R}^{\prime}(x)(h)(t)=2 \int_{0}^{R} \int_{\rho}^{t} x(t+\rho-s) h(s) d s d \eta(\rho), \quad \text { a.e. } t \in(0,1),
$$

for $h \in L_{2}^{\sigma, R}(0,1)$. Further, $F_{R}^{\prime}$ is uniformly Lipschitz in $L_{2}^{\sigma, R}(0,1)$ for all $R>0$ sufficiently small, i.e., there exists some constant $k>0$, such that

$$
\left\|F_{R}^{\prime}\left(x_{1}\right)-F_{R}^{\prime}\left(x_{2}\right)\right\| \leq k\left\|x_{1}-x_{2}\right\|_{\sigma, R}
$$

for $x_{1}, x_{2} \in L_{2}^{\sigma, R}(0,1)$, where $\|\cdot\|$ denotes the usual $\mathcal{L}\left(L_{2}^{\sigma, R}(0,1)\right)$ operator norm.

Proof. Standard arguments (e.g., see [8]) give (2.14). To show the Lipschitz condition, we let $x_{1}, x_{2}, h \in L_{2}^{\sigma, R}(0,1)$ and define

$$
g(t ; R) \equiv 2 \int_{0}^{R} \int_{\rho}^{t}\left(x_{1}(t+\rho-s)-x_{2}(t+\rho-s)\right) h(s) d s d \eta(\rho), \quad \text { a.e. } t \in(0,1) .
$$


Then

$$
\begin{aligned}
& |g(t ; R)| \\
& \leq 2 \int_{0}^{R}\left(\int_{\rho}^{t} e^{2 \sigma \tau} e^{-2 \sigma \tau}\left(x_{1}(\tau)-x_{2}(\tau)\right)^{2} d \tau\right)^{1 / 2}\left(\int_{\rho}^{t} e^{2 \sigma s} e^{-2 \sigma s} h^{2}(s) d s\right)^{1 / 2} d \eta(\rho) \\
& \leq 2 e^{2 \sigma}\left\|x_{1}-x_{2}\right\|_{\sigma, R}\|h\|_{\sigma, R} \int_{0}^{R} d \eta(\rho),
\end{aligned}
$$

for a.e. $t \in(0,1)$. It follows then from Lemma 2.2 that

$$
\left\|F_{R}^{\prime}\left(x_{1}\right)-F_{R}^{\prime}\left(x_{2}\right)\right\| \leq\left(2 \mathcal{C} e^{2 \sigma} \int_{0}^{R} d \eta(\rho)\right)\left\|x_{1}-x_{2}\right\|_{\sigma, R}
$$

Lemma 2.4. Let $v, v_{1}, v_{2} \in \mathcal{B}(0, r) \subseteq L_{2}^{\sigma, R}(0,1)$ and $x \in L_{2}^{\sigma, R}(0,1)$. Then the remainder

$$
\mathcal{R}_{R}(x, v) \equiv F_{R}(x+v)-F_{R}(x)-F_{R}^{\prime}(x) v
$$

of the Fréchet derivative $F_{R}^{\prime}(x)$ satisfies

$$
\left\|\mathcal{R}_{R}(x, v)\right\|_{\sigma, R} \leq e^{2 \sigma} \int_{0}^{R} d \eta(\rho)\|v\|_{\sigma, R}^{2}
$$

and

$$
\begin{aligned}
& \left\|\mathcal{R}_{R}\left(x, v_{1}\right)-\mathcal{R}_{R}\left(x, v_{2}\right)\right\|_{\sigma, R} \\
& \quad \leq 2 e^{2 \sigma} \int_{0}^{R} d \eta(\rho) \max \left\{\left\|v_{1}\right\|_{\sigma, R},\left\|v_{2}\right\|_{\sigma, R}\right\}\left\|v_{1}-v_{2}\right\|_{\sigma, R}
\end{aligned}
$$

for all $R>0$ sufficiently small.

Proof. As in Lemma 1 of [16] and Lemma 2.2 of [8], we may write

$$
\begin{aligned}
\mathcal{R}_{R}(x, v) & =\int_{0}^{1}\left(F_{R}^{\prime}(x+t v)-F_{R}^{\prime}(x)\right) v d t \\
\mathcal{R}_{R}\left(x, v_{1}\right)-\mathcal{R}_{R}\left(x, v_{2}\right) & =\int_{0}^{1}\left(F_{R}^{\prime}\left(x+t v_{1}+(1-t) v_{2}\right)-F_{R}^{\prime}(x)\right)\left(v_{1}-v_{2}\right) d t
\end{aligned}
$$

and then apply the results of Lemma 2.3.

3. Convergence and Well-Posedness Results. Our main convergence results are stated below. They are immediate consequences of Theorem 3.7 and Corollary 3.8 , respectively, which are proved at the end of this section.

TheOREM 3.1. Assume $f^{\delta}$ satisfies the $\mathcal{F}$-data assumption and let $\tau_{\text {data }}=1 / 2$ if $\mathcal{F}=C[0,1+\bar{R}]$ and $\tau_{\text {data }}=2 / 5$ if $\mathcal{F}=L_{2}(0,1+\bar{R})$. Let the measure $\eta$ be given satisfying (2.8)-(2.9).

Then there exists $\bar{C}>0$ and $\kappa_{1}>0$ independent of $R$ such that if the true solution $\bar{x} \in W^{2, \infty}[0,1+\bar{R}]$ of the autoconvolution equation is positive and satisfies

$$
\bar{x}(0)>\bar{C}\left\|\bar{x}^{\prime}\right\|_{\infty}
$$


then for $R=R(\delta)>0$ selected satisfying

$$
\kappa_{1} \delta^{\tau_{\text {data }}} \leq R(\delta) \leq c \delta^{p}
$$

for any $c>0$ and $p \in\left(0, \tau_{\text {data }}\right] \quad\left(c \geq \kappa_{1}\right.$ if $\left.p=\tau_{\text {data }}\right)$ as $\delta \rightarrow 0$, it follows that there is a unique solution $x_{R(\delta)}^{\delta}$ of the regularization equation (2.4) associated with data $f^{\delta}$ which depends continuously on $f^{\delta} \in \mathcal{F}$ and which satisfies

$$
\left\|x_{R(\delta)}^{\delta}-\bar{x}\right\|_{L_{2}(0,1)}=\mathcal{O}\left(\delta^{p}\right)
$$

as $\delta \rightarrow 0$, with optimal rate obtained for $p=\tau_{\text {data }}$. Thus the optimal convergence rate for continuous data is $\mathcal{O}\left(\delta^{1 / 2}\right)$ and the optimal convergence rate for $L_{2}$ data is $\mathcal{O}\left(\delta^{2 / 5}\right)$

Proof. Let $\kappa_{1}=k_{1}^{-\tau_{\text {data }}}$ where $k_{1}$ is defined in the proof of Theorem 3.7 below. Then assumption $(3.2)$ becomes $\delta \leq k_{1} R^{1 /\left(\tau_{\text {data }}\right)}$ and with this assumption Theorem 3.7 gives

$$
\left\|x_{R}^{\delta}-\bar{x}\right\|_{\sigma, R} \leq \hat{C} R
$$

for all $R>0$ sufficiently small. Since

$$
\left\|x_{R}^{\delta}-\bar{x}\right\|_{L_{2}(0,1)}^{2} \leq e^{2 \sigma} \int_{0}^{1} e^{-2 \sigma t}\left|x_{\delta}^{R}-\bar{x}\right|^{2} d x \leq e^{2 \sigma}\left\|x_{R}^{\delta}-\bar{x}\right\|_{\sigma, R}^{2}
$$

the result follows.

Condition (3.1) is an a priori condition or source condition on the true solution $\bar{x}$, a sufficient condition needed to obtain the convergence rate (3.3) in theory. We note that Example 2 in Section 6 below illustrates that this a priori condition is evidently not always needed in practice. The particular nature of this a priori condition reflects the fact that the ill-posedness of the original problem increases as $\bar{x}(0)$ approaches zero; thus if $\bar{x}$ is such that $\bar{x}(0)$ is close to zero, the a priori condition says that convergence is assured provided $\bar{x}$ is relatively "flat". To further explore this restriction, note that for any $y \in W^{1, \infty}(0,1+\bar{R})$,

$$
\frac{\int_{0}^{R} y(t+\rho) d \eta(\rho)}{\int_{0}^{R} d \eta(\rho)}=y(t)\left(1+\mathcal{O}\left(R\left\|y^{\prime}\right\|_{\infty}\right)\right), \text { a.a. } t \in(0,1)
$$

so that the degree to which, for a given value of $R>0$, the measure is able to recover information about $y$ depends to a large extent on the size of $\left\|y^{\prime}\right\|_{\infty}$; i.e., "flat" functions $y$ are handled better under a local averaging process than are functions $y$ with a lot of local variation. Thus, for very ill-posed autoconvolution problems, the source condition says that a method based on local averaging will perform better if the true solution $\bar{x}$ is relatively flat. It's worth noting that a condition like this arises in other areas associated with local regularization; for example, in [4] where a discrepancy principle is developed for the determination of the appropriate value of $R \in(0, \bar{R}]$ in the case of linear Volterra problems, one needs to have the true data $f$ satisfy a similar condition on $\left\|f^{\prime}\right\|_{\infty}$, a condition which is less restrictive if the value of $\bar{R}$ is small and more restrictive if $\bar{R}$ is relatively large.

As the next corollary shows, we may dispense with the source condition (3.1) entirely if we have knowledge of the actual value of $\bar{x}(0)>0$ (or an approximation $\hat{x}(0 ; R)$ to it, as is discussed in Remark 3.3 below), and make use of this information 
in the construction of our measure $\eta$. The proof of this result follows immediately from Corollary 3.8.

Corollary 3.2. Let $\bar{x} \in W^{2, \infty}[0,1+\bar{R}]$ be positive. Assume that the perturbed data $f^{\delta}$ satisfies the $\mathcal{F}$-data assumption and let $\tau_{\text {data }}=1 / 2$ if $\mathcal{F}=C[0,1+\bar{R}]$ and let $\tau_{\text {data }}=2 / 5$ if $\mathcal{F}=L_{2}(0,1+\bar{R})$. Assume that the measure $\eta$ satisfies (2.8)-(2.9) with $\bar{\omega}, \underline{\omega}$ in (2.9) satisfying

$$
\frac{\bar{\omega}}{\underline{\omega}} \leq \tilde{K} \bar{x}(0)
$$

for all $R>0$ sufficiently small, where $\tilde{K}$ is independent of $R$ and $\bar{x}$.

Then there exists $\kappa_{1}>0$ independent of $R$ such that if $R=R(\delta)>0$ is selected satisfying

$$
\kappa_{1} \delta^{\tau_{\text {data }}} \leq R(\delta) \leq c \delta^{p}
$$

for any $c>0$ and $p \in\left(0, \tau_{\text {data }}\right] \quad\left(c \geq \kappa_{1}\right.$ if $\left.p=\tau_{\text {data }}\right)$ as $\delta \rightarrow 0$, it follows that there is a unique solution $x_{R(\delta)}^{\delta}$ of the regularization equation (2.4) associated with data $f^{\delta}$ which depends continuously on $f^{\delta} \in \mathcal{F}$ and which satisfies

$$
\left\|x_{R(\delta)}^{\delta}-\bar{x}\right\|_{L_{2}(0,1)}=\mathcal{O}\left(\delta^{p}\right)
$$

as $\delta \rightarrow 0$, with the optimal rate obtained for $p=\tau_{\text {data }}$. Thus the optimal convergence rate for continuous data is $\mathcal{O}\left(\delta^{1 / 2}\right)$ and the optimal convergence rate for $L_{2}$ data is $\mathcal{O}\left(\delta^{2 / 5}\right)$.

REMARK 3.3. Despite the presence of $\bar{x}(0)$ in condition (3.4) of Corollary 3.2, it is not necessary to know $\bar{x}(0)$ exactly in order to have such a condition hold. It is enough to have some basic information about the size of $\bar{x}$ and to have an approximation $\hat{x}(0 ; R)$ of $\bar{x}(0)$ satisfying

$$
|\bar{x}(0)-\hat{x}(0 ; R)|=\mathcal{O}(R)
$$

as $R \rightarrow 0$. (In the next section we will show that an $\mathcal{O}(R)$ approximation to $\bar{x}(0)$ is easily found.) For example, in the case where it is known that $\bar{x}(0)>1$, then for $\hat{R}$ sufficiently small we have $1<\hat{x}(0, \hat{R})<(\hat{x}(0, \hat{R}))^{2}$, and we may define

$$
\underline{\omega}=\hat{x}(0, \hat{R}), \quad \bar{\omega}=(\hat{x}(0 ; \hat{R}))^{2} .
$$

Then

$$
\frac{\bar{\omega}}{\underline{\omega}}=\hat{x}(0, \hat{R}) \leq 2 \bar{x}(0)
$$

for $\hat{R}$ sufficiently small, so that (3.4) holds with $\tilde{K}=2$. Once $\bar{\omega}$ and $\underline{\omega}$ have been specified, one can always find a suitable $R$-dependent family of measures $\eta$ satisfying (2.8)-(2.9) using these choices of constants; for example, let $\eta$ be given by (2.8) where $\omega(\cdot ; R)$ is the line on $[0, R]$ joining $(0, \underline{\omega})$ to $(R, \bar{\omega})$.

To begin the arguments needed to prove the main convergence results given above, we first rewrite equation (2.2) using similar notation to that used in equation (2.4). That is, the solution $\bar{x}$ of the original autoconvolution equation satisfies

$$
\alpha_{R}(\bar{x}) \bar{x}+F_{R}(\bar{x})=f_{R}+\epsilon_{R},
$$


where

$$
\epsilon_{R}(t) \equiv 2 \int_{0}^{R} \int_{0}^{\rho}(\bar{x}(t)-\bar{x}(t+\rho-s)) \bar{x}(s) d s d \eta(\rho), \quad \text { a.e. } t \in(0,1),
$$

and $\alpha_{R}(\bar{x}), F_{R}(\bar{x})$ are defined as in (2.5)-(2.6), here using $\bar{x}$ instead of $x$, and $f_{R}$ is defined as in (2.7) using $f$ instead of $f^{\delta}$.

For $h \in L_{2}^{\sigma, R}(0,1)$ we define bounded linear operators in $L_{2}^{\sigma, R}(0,1)$,

$$
\begin{aligned}
& B_{R}(\bar{x})(h)(t) \equiv 2 \int_{0}^{R} \int_{0}^{t} \bar{x}(t+\rho-s) h(s) d s d \eta(\rho), \\
& D_{R}(\bar{x})(h)(t) \equiv 2 \int_{0}^{R} \int_{0}^{\rho} \bar{x}(t+\rho-s) h(s) d s d \eta(\rho),
\end{aligned}
$$

for a.e. $t \in(0,1)$ and note that

$$
F_{R}^{\prime}(\bar{x})(h)(t)=B_{R}(\bar{x})(h)(t)-D_{R}(\bar{x})(h)(t), \quad \text { a.e. } t \in(0,1) .
$$

We may then use the expansion of $F_{R}(x)$ in equation (2.4) to write

$$
\alpha_{R}(x) x+F_{R}(\bar{x})+F_{R}^{\prime}(\bar{x})(x-\bar{x})+\mathcal{R}_{R}(\bar{x}, x-\bar{x})=f_{R}^{\delta} .
$$

Combining equations (3.6) and (3.10) with equation (3.11), we obtain

$$
\begin{aligned}
\left(\alpha_{R}(\bar{x}) I+B_{R}(\bar{x})\right)(x-\bar{x})=f_{R}^{\delta}-f_{R}-\epsilon_{R}-\mathcal{R}_{R}(\bar{x}, x-\bar{x}) \\
+D_{R}(\bar{x})(x-\bar{x})+\left(\alpha_{R}(\bar{x})-\alpha_{R}(x)\right) x,
\end{aligned}
$$

where $I$ is the identity operator on $L_{2}^{\sigma, R}(0,1)$. Let us further denote for $v \in L_{2}^{\sigma, R}(0,1)$,

$$
E_{R}(\bar{x}, v)=D_{R}(\bar{x})(v)-\alpha_{R}(v) \bar{x},
$$

where we note that $v \rightarrow E_{R}(\bar{x}, v)$ is linear. Then equation (3.12) becomes

$$
\begin{aligned}
\left(\alpha_{R}(\bar{x}) I+B_{R}(\bar{x})\right)(x-\bar{x})=f_{R}^{\delta} & -f_{R}-\epsilon_{R}-\mathcal{R}_{R}(\bar{x}, x-\bar{x}) \\
& +E_{R}(\bar{x}, x-\bar{x})+\left(\alpha_{R}(x)-\alpha_{R}(\bar{x})\right)(\bar{x}-x) .
\end{aligned}
$$

The following two lemmas will be used to establish the invertibility of the operator $\left(\alpha_{R}(\bar{x}) I+B_{R}(\bar{x})\right) \in \mathcal{L}\left(L_{2}^{\sigma, R}(0,1)\right)$.

Lemma 3.4. If $\bar{x} \in W^{2, \infty}[0,1+\bar{R}]$ and $\bar{x}(t)>0$ for $t \in[0,1+\bar{R}]$, then there exists $\sigma_{0}>0$ independent of $R>0$, such that the operator $B_{R}(\bar{x})$ is accretive in $L_{2}^{\sigma, R}(0,1)$ for $\sigma \geq \sigma_{0}$; i.e.,

$$
\left\langle B_{R}(\bar{x}) v, v\right\rangle_{\sigma, R} \geq 0 \quad \text { for any } \quad v \in L_{2}^{\sigma, R}(0,1),
$$

where $B_{R}$ is defined using a measure $\eta$ satisfying (2.8)-(2.9).

Proof. Following the ideas of [16], only now with $R$-dependent quantities, we make the definition for $t \in[0,1], a_{R}(t) \equiv 2 \int_{0}^{R} \bar{x}(t+\rho) d \eta(\rho)$, so that we may write, for $h \in L_{2}^{\sigma, R}(0,1)$,

$$
B_{R}(\bar{x})(h)(t)=\int_{0}^{t} a_{R}(t-s) h(s) d s, \quad \text { a.e. } t \in(0,1) .
$$


For all $\sigma$ sufficiently large, we will first show that the operator $B_{R}^{\sigma}(\bar{x})$ is accretive in $L_{2}(0,1)$, where $B_{R}^{\sigma}(\bar{x})$ is defined as in (3.15) except that the kernel $a_{R}(t)$ for $B_{R}$ is replaced by $a_{R}[\sigma](t)=e^{-\sigma t} a_{R}(t)$. Defining

$$
A_{0}=\min _{t \in[0,1+\bar{R}]} \bar{x}(t)>0, \quad A_{1}=\left\|\bar{x}^{\prime}\right\|_{L^{\infty}[0,1+\bar{R}]}, \quad A_{2}=\left\|\bar{x}^{\prime \prime}\right\|_{L^{\infty}[0,1+\bar{R}]},
$$

then

$$
\begin{gathered}
\min _{t \in[0,1]} a_{R}(t) \geq 2 A_{0} \int_{0}^{R} d \eta(\rho), \\
\left\|a_{R}^{\prime}\right\|_{L^{\infty}[0,1]} \leq 2 A_{1} \int_{0}^{R} d \eta(\rho), \\
\left\|a_{R}^{\prime \prime}\right\|_{L^{\infty}[0,1]} \leq 2 A_{2} \int_{0}^{R} d \eta(\rho),
\end{gathered}
$$

and for a.e. $t \in(0,1)$,

$$
\begin{aligned}
& a_{R}[\sigma](t) \geq e^{-\sigma t}\left(2 A_{0} \int_{0}^{R} d \eta(\rho)\right), \\
& a_{R}^{\prime}[\sigma](t) \leq e^{-\sigma t} 2\left(-\sigma A_{0}+A_{1}\right) \int_{0}^{R} d \eta(\rho), \\
& a_{R}^{\prime \prime}[\sigma](t) \geq e^{-\sigma t} 2\left(\sigma^{2} A_{0}-2 \sigma A_{1}-A_{2}\right) \int_{0}^{R} d \eta(\rho) .
\end{aligned}
$$

For $\sigma \geq \sigma_{0}$, where

$$
\sigma_{0} \geq \frac{A_{1}+\sqrt{A_{1}^{2}+A_{0} A_{2}}}{A_{0}}, \quad(\text { independent of } R \text { ) }
$$

we then have from the positivity of $\eta$ that

$$
a_{R}[\sigma](t) \geq 0, \quad a_{R}[\sigma]^{\prime}(t) \leq 0, \quad a_{R}[\sigma]^{\prime \prime}(t) \geq 0,
$$

for a.e. $t \in(0,1)$.

Therefore, the kernel $a_{R}[\sigma](\cdot)$ is nonnegative, nonincreasing and convex. Using Lemma 2 of [16] for $\sigma \geq \sigma_{0}$, we have $B_{R}^{\sigma}(\bar{x})$ accretive on $L_{2}(0,1)$, from which it follows that $B_{R}(\bar{x})$ is accretive in $L_{2}(0,1)$ with a weighted $e^{-2 \sigma t}$-norm. A similar statement may be made for $B_{R}(\bar{x})$ using a weighted $L_{2}(0, R)$ norm, from which it follows that $B_{R}(\bar{x})$ is accretive in $L_{2}^{\sigma, R}(0,1)$ for all $\sigma \geq \sigma_{0}$.

LEMmA 3.5. Let $\bar{x} \in C^{1}[0,1+\bar{R}]$ satisfy $\bar{x}(0)>0$ and assume that the measure $\eta$ satisfies (2.8)-(2.9). Then for $R>0$ sufficiently small we have $\alpha_{R}(\bar{x})>0$ and

$$
\frac{1}{\alpha_{R}(\bar{x})} \leq \frac{1}{\bar{x}(0) \int_{0}^{R} \rho d \eta(\rho)} .
$$

Proof. We can write

$$
\alpha_{R}(\bar{x})=2 \bar{x}(0) \int_{0}^{R} \rho d \eta(\rho)[1+h(R)],
$$


where

$$
h(R)=\frac{1}{\bar{x}(0) \int_{0}^{R} \rho d \eta(\rho)} \int_{0}^{R} \int_{0}^{\rho} s \bar{x}^{\prime}(\xi(s)) d s d \eta(\rho) .
$$

for some $\xi(s) \in[0, R]$. But

$$
|h(R)| \leq \frac{1}{\bar{x}(0) \int_{0}^{R} \rho d \eta(\rho)}\left\|\bar{x}^{\prime}\right\|_{\infty} \int_{0}^{R} \frac{\rho^{2}}{2} d \eta(\rho) \leq \frac{\left\|\bar{x}^{\prime}\right\|_{\infty} K(2)}{2 \bar{x}(0)} R,
$$

where $K(2)$ is defined in (2.10), so it follows that

$$
\frac{1}{\alpha_{R}(\bar{x})}=\frac{1}{2 \bar{x}(0) \int_{0}^{R} \rho d \eta(\rho)}(1+\mathcal{O}(R)) \quad \text { as } R \rightarrow 0 .
$$

As a consequence of Lemmas 3.4 and 3.5, we have that if $\bar{x} \in W^{2, \infty}[0,1+\bar{R}]$ with $\bar{x}(0)>0$, then $\alpha_{R}(\bar{x})>0$ and $\left(\alpha_{R}(\bar{x}) I+B_{R}(\bar{x})\right)^{-1} \in \mathcal{L}\left(L_{2}^{\sigma, R}(0,1)\right)$ for all $R>0$ sufficiently small; in addition, we have the following estimate (see [33]):

$$
\left\|\left(\alpha_{R}(\bar{x}) I+B_{R}(\bar{x})\right)^{-1}\right\| \leq \frac{1}{\alpha_{R}(\bar{x})},
$$

for $\|\cdot\|$ the $\mathcal{L}\left(L_{2}^{\sigma, R}(0,1)\right)$ operator norm, and for $\sigma \geq \sigma_{0}$, where $\sigma_{0}>0$ is independent of $R$.

We are now ready to rewrite our regularized equation (2.4) as

$$
x=H_{R} x,
$$

where from (3.14), $H_{R}: L_{2}^{\sigma, R}(0,1) \mapsto L_{2}^{\sigma, R}(0,1)$ is given by

$$
\begin{aligned}
H_{R} x=\left(\alpha_{R}(\bar{x}) I+B_{R}(\bar{x})\right)^{-1}\left[f_{R}^{\delta}-f_{R}-\epsilon_{R}-\mathcal{R}_{R}(\bar{x}, x-\bar{x})\right. \\
\left.+E_{R}(\bar{x}, x-\bar{x})+\left(\alpha_{R}(x)-\alpha_{R}(\bar{x})\right)(\bar{x}-x)\right]+\bar{x} .
\end{aligned}
$$

In the next lemma we bound relevant quantities on the right hand side of equation (3.19).

Lemma 3.6. Let $\bar{x} \in C^{1}[0,1+\bar{R}]$ satisfy $\bar{x}(0)>0$, let $f^{\delta}$ satisfy the $\mathcal{F}$-data assumption, assume the measure $\eta$ satisfies (2.8)-(2.9), and let $\sigma>0$. Let $x_{1}, x_{2} \in$ $L_{2}^{\sigma, R}(0,1)$. Then

$$
\begin{gathered}
\left\|f_{R}^{\delta}-f_{R}\right\|_{\sigma, R} \leq \begin{cases}\delta \mathcal{C} \bar{\omega} R, & \text { if } \mathcal{F}=C[0,1+\bar{R}], \\
\delta \mathcal{C} \bar{\omega} R^{1 / 2}, & \text { if } \mathcal{F}=L_{2}(0,1+\bar{R}),\end{cases} \\
\left\|E_{R}\left(\bar{x}, x_{1}-x_{2}\right)\right\|_{\sigma, R} \leq \mathcal{C} \frac{2 e^{\sigma, R}}{\sqrt{3}}\left\|\bar{x}^{\prime}\right\|_{\infty}\left\|x_{1}-x_{2}\right\|_{\sigma, R} \int_{0}^{R} \rho^{3 / 2} d \eta(\rho), \\
\left\|\epsilon_{R}\right\|_{\sigma, R} \leq 2 \mathcal{C} \sqrt{\frac{2}{3}}\left\|\bar{x}^{\prime}\right\|_{\infty}\left(\bar{x}(0) \int_{0}^{R} \rho^{2} d \eta(\rho)+\frac{\left\|\bar{x}^{\prime}\right\|_{\infty}}{\sqrt{3}} \int_{0}^{R} \rho^{3} d \eta(\rho)\right),
\end{gathered}
$$


and

$$
\left|\alpha_{R}\left(x_{1}\right)-\alpha_{R}\left(x_{2}\right)\right| \leq 2 e^{\sigma R} \sqrt{\frac{R}{\mathcal{C}^{2}-1}}\left\|x_{1}-x_{2}\right\|_{\sigma, R} \int_{0}^{R} \rho^{1 / 2} d \eta(\rho) .
$$

Proof. The bounds in (3.20)-(3.22) each follow from applications of Lemma 2.2 using obvious choices of the function $g=g(\cdot ; R)$. Inequality $(3.20)$ is straightforward in the case of $f^{\delta} \in C[0,1+\bar{R}]$ since

$$
\begin{aligned}
\left|f_{R}^{\delta}(t)-f_{R}(t)\right| & =\left|\int_{0}^{R}\left(f^{\delta}(t+\rho)-f(t+\rho)\right) \omega(\rho ; R) d \rho\right| \\
& \leq \delta \bar{\omega} R .
\end{aligned}
$$

For the case of $f^{\delta} \in L_{2}(0,1+\bar{R}]$ we observe that $f_{R}^{\delta}-f_{R} \in L_{\infty}(0,1)$ since

$$
\begin{aligned}
\left|f_{R}^{\delta}(t)-f_{R}(t)\right| & \leq\left(\int_{0}^{R}\left|f^{\delta}(t+\rho)-f(t+\rho)\right|^{2} d \rho\right)^{1 / 2}\left(\int_{0}^{R}|\omega(\rho ; R)|^{2} d \rho\right)^{1 / 2} \\
& \leq\left\|f^{\delta}-f\right\|_{L_{2}(0,1+\bar{R})} \bar{\omega} R^{1 / 2}
\end{aligned}
$$

for a.a. $t \in(0,1+\bar{R})$. The result then follows from Lemma 2.2 .

For $(3.21)$ we use the fact that for a.e. $t \in(0,1)$,

$$
\begin{aligned}
& \left|E_{R}\left(\bar{x}, x_{1}-x_{2}\right)(t)\right| \\
& \leq 2 \int_{0}^{R} \int_{0}^{\rho}|\bar{x}(t+\rho-s)-\bar{x}(t)|\left|x_{1}(s)-x_{2}(s)\right| d s d \eta(\rho) \\
& \leq 2\left\|\bar{x}^{\prime}\right\|_{\infty} \int_{0}^{R}\left(\int_{0}^{\rho}(\rho-s)^{2} d s\right)^{1 / 2}\left(\int_{0}^{\rho} e^{2 \sigma s} e^{-2 \sigma s}\left|x_{1}(s)-x_{2}(s)\right|^{2} d s\right)^{1 / 2} d \eta(\rho) .
\end{aligned}
$$

For (3.22) we observe that for some $\xi \in(0, R)$,

$$
\begin{aligned}
\left|\epsilon_{R}(t)\right| & \leq 2\left\|\bar{x}^{\prime}\right\|_{\infty} \int_{0}^{R}\left(\int_{0}^{\rho}(\rho-s)^{2} d s\right)^{1 / 2}\left(\int_{0}^{\rho}\left(\bar{x}(0) d s+\bar{x}^{\prime}(\xi) s\right)^{2} d s\right)^{1 / 2} d \eta(\rho) \\
& \leq 2\left\|\bar{x}^{\prime}\right\|_{\infty} \int_{0}^{R}\left[\frac{\rho^{3}}{3} 2\left(\bar{x}(0)^{2} \rho+\left\|\bar{x}^{\prime}\right\|_{\infty}^{2} \frac{\rho^{3}}{3}\right)\right]^{1 / 2} d \eta(\rho) \\
& \leq 2\left\|\bar{x}^{\prime}\right\|_{\infty} \int_{0}^{R} \sqrt{\frac{2}{3}} \rho^{3 / 2}\left(\bar{x}(0) \rho^{1 / 2}+\left\|\bar{x}^{\prime}\right\|_{\infty} \frac{\rho^{3 / 2}}{\sqrt{3}}\right) d \eta(\rho)
\end{aligned}
$$

for a.e. $t \in(0,1)$, from which the bound in (3.22) follows.

Finally, using the fact that

$$
\begin{aligned}
\left|\alpha_{R}\left(x_{1}\right)-\alpha_{R}\left(x_{2}\right)\right| & \leq 2 \int_{0}^{R}\left|\int_{0}^{\rho}\left(x_{1}(s)-x_{2}(s)\right) d s\right| d \eta(\rho) \\
& \leq 2 e^{\sigma R}\left(\int_{0}^{R} e^{-2 \sigma t}\left|x_{1}(t)-x_{2}(t)\right|^{2} d t\right)^{1 / 2} \int_{0}^{R} \rho^{1 / 2} d \eta(\rho),
\end{aligned}
$$

the estimate in (3.23) is obtained. 
We are now ready to state our main convergence results which are given below in Theorem 3.7 and Corollary 3.8.

Theorem 3.7. Assume that $f^{\delta}$ satisfies the $\mathcal{F}$-data condition and let $\tau_{\text {data }}=1 / 2$ in the case of $\mathcal{F}=C[0,1+\bar{R}]$ and let $\tau_{\text {data }}=2 / 5$ in the case of $\mathcal{F}=L_{2}(0,1+\bar{R})$. Assume the measure $\eta(\rho)>0$ satisfies (2.8)-(2.9) and that the autoconvolution problem (1.1) has a positive solution $\bar{x} \in W^{2, \infty}[0,1+\bar{R}]$ satisfying

$$
\bar{x}(0)>9 b^{2} e^{2 \sigma}\left\|\bar{x}^{\prime}\right\|_{\infty}
$$

for $\sigma \geq \sigma_{0}$ and $b \geq 2 \bar{\omega} / \underline{\omega}$, where $\bar{\omega}, \underline{\omega}$ are given in (2.8). Then for any $\mathcal{C} \in(1,9 / 8]$, there exist constants $k_{1}>0$ and $\hat{C}=\hat{C}(\mathcal{C})>0$ independent of $R$ such that if $\delta=$ $\delta(R)>0$ satisfies

$$
\delta \leq k_{1} R^{1 / \tau_{\text {data }}}
$$

then for all $R>0$ sufficiently small the regularized equation (3.18) has a unique solution $x_{R}^{\delta} \in L_{2}^{\sigma, R}(0,1)$ satisfying

$$
\left\|x_{R}^{\delta}-\bar{x}\right\|_{\sigma, R}^{2} \leq \hat{C}^{2} R^{2}
$$

where $\|\cdot\|_{\sigma, R}$ is defined from (2.11) using the value of $\mathcal{C}$ given above. Further, $x_{R}^{\delta} \in$ $L_{2}^{\sigma, R}(0,1)$ depends continuously on $f^{\delta} \in \mathcal{F}$ for all $R>0$ sufficiently small.

Proof. We will apply the contraction mapping principle to the regularized equation (3.18) in the ball $\mathcal{B}(\bar{x}, \hat{C} R)$. From Lemmas 2.4, 3.5, 3.6, and inequality (3.17) we have

$$
\begin{aligned}
\left\|H_{R} x-\bar{x}\right\|_{\sigma, R} \leq & \frac{1}{\alpha(\bar{x})}\left\|f_{R}^{\delta}-f_{R}\right\|_{\sigma, R}+\frac{1}{\alpha(\bar{x})}\left\|\mathcal{R}_{R}(\bar{x}, x-\bar{x})\right\|_{\sigma, R}+\frac{1}{\alpha(\bar{x})}\left\|\epsilon_{R}\right\|_{\sigma, R} \\
& +\frac{1}{\alpha(\bar{x})}\left\|E_{R}(\bar{x}, x-\bar{x})\right\|_{\sigma, R}+\frac{\left|\alpha_{R}(x)-\alpha_{R}(\bar{x})\right|}{\alpha_{R}(\bar{x})}\|x-\bar{x}\|_{\sigma, R} \\
\leq & \frac{\delta \mathcal{C}}{\bar{x}(0)} \frac{2 \bar{\omega}}{\underline{\omega}} R^{p}+\frac{e^{2 \sigma}}{\bar{x}(0)} \frac{2 \bar{\omega}}{\underline{\omega}} R^{-1}\|x-\bar{x}\|_{\sigma, R}^{2} \\
+ & 2 \mathcal{C} \sqrt{\frac{2}{3}}\left\|\bar{x}^{\prime}\right\|_{\infty}\left(\frac{2 \bar{\omega}}{3 \underline{\omega}} R+\frac{\left\|\bar{x}^{\prime}\right\|_{\infty}}{\sqrt{3} \bar{x}(0)} K(3) R^{2}\right) \\
& +\frac{2 \mathcal{C}\left\|\bar{x}^{\prime}\right\|_{\infty} e^{\sigma R}}{\sqrt{3} \bar{x}(0)} K\left(\frac{3}{2}\right) R^{1 / 2}\|x-\bar{x}\|_{\sigma, R} \\
+ & \frac{2 e^{\sigma R}}{\bar{x}(0)} K\left(\frac{1}{2}\right) R^{-1 / 2}\|x-\bar{x}\|_{\sigma, R}^{2} \sqrt{\frac{R}{\mathcal{C}^{2}-1}}
\end{aligned}
$$

for $\sigma \geq \sigma_{0}$, and where $p=-1$ for $\mathcal{F}=C[0,1+\bar{R}]$ and $p=-3 / 2$ for $\mathcal{F}=L_{2}(0,1+\bar{R})$. Using assumption (3.25) and the fact that $\|x-\bar{x}\|_{\sigma, R} \leq \hat{C} R$, we have

$$
\begin{aligned}
\| H_{R} x- & \bar{x}\left\|_{\sigma, R} \leq \frac{k_{1} \mathcal{C}}{\bar{x}(0)} \frac{2 \bar{\omega}}{\underline{\omega}} R+\frac{e^{2 \sigma}}{\bar{x}(0)} \frac{2 \bar{\omega}}{\underline{\omega}} \hat{C}^{2} R+2 \mathcal{C} \sqrt{\frac{2}{3}}\right\| \bar{x}^{\prime} \|_{\infty} \frac{2 \bar{\omega}}{3 \underline{\omega}} R \\
& +\frac{2 \sqrt{2} \mathcal{C}\left\|\bar{x}^{\prime}\right\|_{\infty}^{2}}{3 \bar{x}(0)} K(3) R^{2}+\frac{2 \mathcal{C}\left\|\bar{x}^{\prime}\right\|_{\infty} e^{\sigma R}}{\sqrt{3} \bar{x}(0)} K\left(\frac{3}{2}\right) \hat{C} R^{\frac{3}{2}}+\frac{2 e^{\sigma R} K\left(\frac{1}{2}\right)}{\bar{x}(0)} \frac{\hat{C}^{2} R^{2}}{\sqrt{\mathcal{C}^{2}-1}}
\end{aligned}
$$


Therefore to have $\left\|H_{R} x-\bar{x}\right\|_{\sigma, R} \leq \hat{C} R$ for some $\hat{C}>0$ and all $R>0$ sufficiently small, a sufficient condition is that

$$
\frac{1}{\bar{x}(0)} b k_{1} \mathcal{C}+\left\|\bar{x}^{\prime}\right\|_{\infty} b \mathcal{C}+\frac{e^{2 \sigma}}{\bar{x}(0)} b \hat{C}^{2}<\hat{C} .
$$

If we let

$$
k_{1}=\left\|\bar{x}^{\prime}\right\|_{\infty} \bar{x}(0),
$$

then equation (3.26) then becomes

$$
L(\hat{C}) \equiv \frac{e^{2 \sigma} b}{\bar{x}(0)} \hat{C}^{2}-\hat{C}+2 b \mathcal{C}\left\|\bar{x}^{\prime}\right\|_{\infty}<0 .
$$

It is not hard to see $L(\hat{C})=0$ has two distinct positive solutions $0<\hat{C}_{1}<\hat{C}_{2}$ by assumption (3.24) when $\mathcal{C} \in(1,9 / 8]$. Then for $\hat{C}$ satisfying $\hat{C}_{1}<\hat{C}<\hat{C}_{2}$, we have $L(\hat{C})<0$, thus $\left\|H_{R} x-\bar{x}\right\|_{\sigma, R} \leq \hat{C} R$ for all $R>0$ sufficiently small.

To further demonstrate that $H_{R}$ is a contraction on $\mathcal{B}(\bar{x}, \hat{C} R)$, we let $x_{1}, x_{2} \in$ $\mathcal{B}(\bar{x}, \hat{C} R)$ and note that

$$
\begin{aligned}
& \left\|H_{R} x_{1}-H_{R} x_{2}\right\|_{\sigma, R} \\
& =\|\left(\alpha_{R}(\bar{x}) I+B_{R}(\bar{x})\right)^{-1}\left\{\mathcal{R}_{R}\left(\bar{x}, x_{2}-\bar{x}\right)-\mathcal{R}_{R}\left(\bar{x}, x_{1}-\bar{x}\right)+E_{R}\left(\bar{x}, x_{1}-x_{2}\right)\right. \\
& \left.\quad \quad-\left[\left(\alpha_{R}\left(x_{1}\right)-\alpha_{R}(\bar{x})\right)\left(x_{1}-\bar{x}\right)-\left(\alpha_{R}\left(x_{2}\right)-\alpha_{R}(\bar{x})\right)\left(x_{2}-\bar{x}\right)\right]\right\} \|_{\sigma, R} \\
& \leq T_{R}\left(x_{1}, x_{2}\right),
\end{aligned}
$$

where

$$
\begin{aligned}
T_{R}\left(x_{1}, x_{2}\right) \equiv & \frac{1}{\alpha_{R}(\bar{x})}\left\|\mathcal{R}_{R}\left(\bar{x}, x_{2}-\bar{x}\right)-\mathcal{R}_{R}\left(\bar{x}, x_{1}-\bar{x}\right)\right\|_{\sigma, R}+\frac{1}{\alpha_{R}(\bar{x})}\left\|E_{R}\left(\bar{x}, x_{1}-x_{2}\right)\right\|_{\sigma, R} \\
& \quad+\frac{1}{\alpha_{R}(\bar{x})}\left\|\left(\alpha_{R}\left(x_{1}\right)-\alpha_{R}(\bar{x})\right)\left(x_{1}-\bar{x}\right)-\left(\alpha_{R}\left(x_{2}\right)-\alpha_{R}(\bar{x})\right)\left(x_{2}-\bar{x}\right)\right\|_{\sigma, R} .
\end{aligned}
$$

Since

$$
\begin{aligned}
& \frac{1}{\alpha_{R}(\bar{x})}\left\|\left(\alpha_{R}\left(x_{1}\right)-\alpha_{R}(\bar{x})\right)\left(x_{1}-\bar{x}\right)-\left(\alpha_{R}\left(x_{2}\right)-\alpha_{R}(\bar{x})\right)\left(x_{2}-\bar{x}\right)\right\|_{\sigma, R} \\
& =\frac{1}{\alpha_{R}(\bar{x})}\left\|\left(\alpha_{R}\left(x_{1}\right)-\alpha_{R}\left(x_{2}\right)\right)\left(x_{1}-\bar{x}\right)+\left(\alpha_{R}\left(x_{2}\right)-\alpha_{R}(\bar{x})\right)\left(x_{1}-x_{2}\right)\right\|_{\sigma, R} \\
& \leq \frac{\left|\alpha_{R}\left(x_{1}\right)-\alpha_{R}\left(x_{2}\right)\right|}{\alpha(\bar{x})}\left\|x_{1}-\bar{x}\right\|_{\sigma, R}+\frac{\left|\alpha_{R}\left(x_{2}\right)-\alpha_{R}(\bar{x})\right|}{\alpha(\bar{x})}\left\|x_{1}-x_{2}\right\|_{\sigma, R} \\
& \leq \frac{2 e^{\sigma R} \int_{0}^{R} \rho^{\frac{1}{2}} d \eta(\rho)}{\bar{x}(0) \int_{0}^{R} \rho d \eta(\rho)} \sqrt{\frac{R}{\mathcal{C}^{2}-1}}\left\{\left\|x_{1}-x_{2}\right\|_{\sigma, R}\left\|x_{1}-\bar{x}\right\|_{\sigma, R}+\left\|x_{2}-\bar{x}\right\|_{\sigma, R}\left\|x_{1}-x_{2}\right\|_{\sigma, R}\right\} \\
& \leq \frac{4 e^{\sigma R} K\left(\frac{1}{2}\right) \hat{C} R}{\bar{x}(0) \sqrt{\mathcal{C}^{2}-1}}\left\|x_{1}-x_{2}\right\|_{\sigma, R},
\end{aligned}
$$

we have

$$
\begin{aligned}
& T_{R}\left(x_{1}, x_{2}\right) \\
& \leq\left[\frac{2 e^{2 \sigma}}{\bar{x}(0)} \frac{2 \bar{\omega}}{\underline{\omega}} \hat{C}\right]\left\|x_{1}-x_{2}\right\|_{\sigma, R}+\left[\frac{2 \mathcal{C}\left\|\bar{x}^{\prime}\right\|_{\infty} e^{\sigma R}}{\sqrt{3} \bar{x}(0)} K\left(\frac{3}{2}\right) R^{\frac{1}{2}}+\frac{4 e^{\sigma R} K\left(\frac{1}{2}\right) \hat{C} R}{\bar{x}(0) \sqrt{\mathcal{C}^{2}-1}}\right]\left\|x_{1}-x_{2}\right\|_{\sigma, R} .
\end{aligned}
$$


Thus if we require that

$$
\hat{C}<\frac{\bar{x}(0)}{2 e^{2 \sigma} b}
$$

it follows that

$$
\hat{C}<\frac{\bar{x}(0)}{2 e^{2 \sigma}} \frac{\underline{\omega}}{2 \bar{\omega}}
$$

and for $R>0$ sufficiently small,

$$
\left\|H_{R} x_{1}-H_{R} x_{2}\right\|_{\sigma, R} \leq T_{R}\left(x_{1}, x_{2}\right) \leq q\left\|x_{1}-x_{2}\right\|_{\sigma, R}
$$

for any $x_{1}, x_{2} \in \mathcal{B}(\bar{x}, \hat{C} R)$ with some $q<1$. Further we note that $\frac{\hat{C}_{1}+\hat{C}_{2}}{2}=\frac{\bar{x}(0)}{2 e^{2 \sigma} b}$, therefore our regularized equation (3.18) has a unique solution $x_{R}^{\delta}$ in $\mathcal{B}(\bar{x}, \hat{C} R)$ for $\hat{C}$ satisfying $\hat{C}_{1}<\hat{C}<\frac{\bar{x}(0)}{2 e^{2 \sigma} b}$.

Finally, fix $R>0$ sufficiently small and let $f_{1}^{\delta}, f_{2}^{\delta} \in \mathcal{F}$ satisfy

$$
\left\|f_{i}^{\delta}-f\right\|_{\mathcal{F}} \leq \delta \leq k_{1} R^{1 / \tau_{\text {data }}}, \quad i=1,2,
$$

for the value of $k_{1}$ given in (3.27), and where $\mathcal{F}$ is either $C[0,1+\bar{R}]$ or $L_{2}(0,1+\bar{R})$. Let $\hat{C}, \mathcal{C}, \sigma$, and $q$ be as specified above and let $f_{R, i}^{\delta}$ and $H_{R, i}$ be defined for $i=1,2$ as in (2.7) and (3.19), respectively, using $f_{i}^{\delta}$ instead of $f^{\delta}$ as data. Then for $i=1,2$ there exists a unique solution $x_{R, i}^{\delta} \in \mathcal{B}(\bar{x}, \hat{C} R) \subset L_{2}^{\sigma, R}(0,1)$ of the equation $x=H_{R, i} x$. Further,

$$
\begin{aligned}
& \left\|x_{R, 1}^{\delta}-x_{R, 2}^{\delta}\right\|_{\sigma, R}=\left\|H_{R, 1} x_{R, 1}^{\delta}-H_{R, 2} x_{R, 2}^{\delta}\right\|_{\sigma, R} \\
& =\|\left(\alpha_{R}(\bar{x}) I+B_{R}(\bar{x})\right)^{-1}\left\{\left(f_{R, 1}^{\delta}-f_{R, 2}^{\delta}\right)\right. \\
& +\mathcal{R}_{R}\left(\bar{x}, x_{R, 2}^{\delta}-\bar{x}\right)-\mathcal{R}_{R}\left(\bar{x}, x_{R, 1}^{\delta}-\bar{x}\right)+E_{R}\left(\bar{x}, x_{R, 1}^{\delta}-x_{R, 2}^{\delta}\right) \\
& \left.-\left[\left(\alpha_{R}\left(x_{R, 1}^{\delta}\right)-\alpha_{R}(\bar{x})\right)\left(x_{R, 1}^{\delta}-\bar{x}\right)-\left(\alpha_{R}\left(x_{R, 2}^{\delta}\right)-\alpha_{R}(\bar{x})\right)\left(x_{R, 2}^{\delta}-\bar{x}\right)\right]\right\} \|_{\sigma, R} \\
& \leq \frac{1}{\alpha_{R}(\bar{x})}\left\|f_{R, 1}^{\delta}-f_{R, 2}^{\delta}\right\|_{\sigma, R}+T_{R}\left(x_{R, 1}^{\delta}, x_{R, 2}^{\delta}\right) \\
& \leq \frac{\mathcal{C}\left\|f_{1}^{\delta}-f_{2}^{\delta}\right\|_{\mathcal{F}}}{\bar{x}(0)} \frac{2 \bar{\omega}}{\underline{\omega}} R^{p}+q\left\|x_{R, 1}^{\delta}-x_{R, 2}^{\delta}\right\|_{\sigma, R},
\end{aligned}
$$

where $p=-1$ in the case of $\mathcal{F}=C[0,1+\bar{R}]$ and $p=-3 / 2$ in the case of $\mathcal{F}=$ $L_{2}(0,1+\bar{R})$, and where we have used arguments like those needed to verify (3.20) in the first term above. Therefore

$$
\left\|x_{R, 1}^{\delta}-x_{R, 2}^{\delta}\right\|_{\sigma, R} \leq \frac{\mathcal{C} b}{(1-q) \bar{x}(0)} R^{p}\left\|f_{1}^{\delta}-f_{2}^{\delta}\right\|_{\mathcal{F}}
$$

and continuous dependence of solutions on data is obtained for the regularized equation (3.18).

$\square$

COROLlary 3.8. Assume that the autoconvolution problem (1.1) has a positive solution $\bar{x} \in W^{2, \infty}[0,1+\bar{R}]$ and that the measure $\eta$ satisfies (2.8)-(2.9) with constants $\bar{\omega}, \underline{\omega}$ in (2.9) satisfying (3.4) for all $R>0$ sufficiently small, where $\tilde{K}$ is independent 
of $R$ and $\bar{x}$. Let $f^{\delta}$ satisfy the $\mathcal{F}$-data condition and let $\tau_{\text {data }}=1 / 2$ in the case of $\mathcal{F}=C[0,1+\bar{R}]$ and let $\tau_{\text {data }}=2 / 5$ in the case of $\mathcal{F}=L_{2}(0,1+\bar{R})$.

Then there exist constants $k_{1}, \hat{C}>0$ independent of $R$ such that if $\delta=\delta(R)>0$ satisfies

$$
\delta \leq k_{1} R^{1 / \tau_{\text {data }}}
$$

then for all $R>0$ sufficiently small the regularized equation (3.18) has a unique solution $x_{R}^{\delta} \in L_{2}^{\sigma, R}(0,1)$ satisfying

$$
\left\|x_{R}^{\delta}-\bar{x}\right\|_{\sigma, R}^{2} \leq \hat{C}^{2} R^{2}
$$

where $\|\cdot\|_{\sigma, R}$ is defined from (2.11) using the value of $\mathcal{C}=\sqrt{2}$. Further, $x_{R}^{\delta} \in$ $L_{2}^{\sigma, R}(0,1)$ depends continuously on $f^{\delta} \in \mathcal{F}$ for all $R>0$ sufficiently small.

Proof. Let $\mathcal{C}=\sqrt{2}$ and define $\tilde{b}=2 \tilde{K}$ where $\tilde{K}$, given by (3.4), is independent of $R$ and $\bar{x}$. We observe that without loss of generality we may assume that $\bar{x}$ satisfies

$$
\bar{x}(0)\left\|\bar{x}^{\prime}\right\|_{\infty}<\frac{\sqrt{2} e^{-2 \sigma}}{16 \tilde{b}^{2}}
$$

because if this does not already hold, we may rescale equation (1.1) via division by some $\kappa^{2}>1$ to obtain a new equation with data $f / \kappa^{2}$ and solution $\bar{y}=\bar{x} / \kappa$, where $\bar{y}$ satisfies (3.32). Note that a similar rescaling of $\eta$ will not change the value of $\tilde{K}$, i.e., of $\tilde{b}$.

The proof of the corollary follows the proof of Theorem 3.7 with only a few changes. First we note that if we let $b=\tilde{b} \bar{x}(0)$, then $b \geq 2 \bar{\omega} / \underline{\omega}$ and equation $(3.26)$ still holds from the proof of Theorem 3.7. Writing (3.26) in terms of $\tilde{b}$, we then have the following sufficient condition ensuring that $\left\|H_{R} x-\bar{x}\right\|_{\sigma, R}<\hat{C} R$ for some $\hat{C}>0$ :

$$
k_{1} \tilde{b} \mathcal{C}+\left\|\bar{x}^{\prime}\right\|_{\infty} \bar{x}(0) \tilde{b} \mathcal{C}+e^{2 \sigma} \tilde{b} \hat{C}^{2}<\hat{C} .
$$

Defining $k_{1}$ as in (3.27), the inequality in (3.33) now becomes

$$
L(\hat{C})=e^{2 \sigma} \tilde{b} \hat{C}^{2}-\hat{C}+2\left\|\bar{x}^{\prime}\right\|_{\infty} \bar{x}(0) \tilde{b} \mathcal{C}<0 .
$$

Under condition (3.32) the quadratic $L$ has two distinct positive roots $0<\hat{C}_{1}<\hat{C}_{2}$ so that we may pick $\hat{C} \in\left(\hat{C}_{1}, \hat{C}_{2}\right)$ to guarantee that $L(\hat{C})<0$.

The remainder of the proof of Theorem 3.7 carries over without change; we note only that the new condition (3.30) on $\hat{C}$ in that proof creates no difficulty because here our roots $\hat{C}_{1}$ and $\hat{C}_{2}$ satisfy $\frac{\hat{C}_{1}+\hat{C}_{2}}{2}=\frac{1}{2 e^{2 \sigma} \tilde{b}}=\frac{\bar{x}(0)}{2 e^{2 \sigma b}}$ as needed for the proof of Theorem 3.7.

4. Discretization. In this section, we will consider a discretized version of the regularized equation $(2.4)$, which leads to a stable method on the interval $[0,1]$; the resulting method is nonlinear and non-sequential on the initial small interval $[0, R]$, but sequential and linear on the larger interval $[R, 1]$.

We will consider a collocation-based discretization of (2.4) where, for simplicity, we will assume that the measure $\eta$ is given by the Lebesgue measure, i.e.,

$$
\int_{0}^{R} g(\rho) d \eta(\rho)=\int_{0}^{R} g(\rho) d \rho,
$$


and note that condition (2.9) is satisfied in this case. Indeed our numerical results were so good using this simple measure that there seemed little need to construct a weighted measure based on an approximate value of $\bar{x}(0)$ as was discussed as a possibility in Remark 3.3.

Let $N=1,2,3, \ldots$ and define

$$
t_{i}=i \Delta t, \quad i=0,1, \ldots, N, \quad \Delta t=1 / N .
$$

We will also let $R=r \Delta t$, where $r \in\{1,2, \ldots, N\}$ is fixed $(r \ll N$, in general).

For $i=2,3, \ldots, N$, let $\chi_{i}(t)$ be the indicator function on the interval $\left(t_{i-1}, t_{i}\right]$ and $\chi_{1}(t)$ the indicator function on the interval $\left[t_{0}, t_{1}\right]$, and define the approximation space of piecewise constant functions on $[0,1]$ by $\mathcal{S}_{N}=\operatorname{span}\left\{\chi_{i}, i=1,2, \ldots, N\right\}$. We then seek $x \in \mathcal{S}_{N}$ satisfying the collocation equations

$$
2 \int_{0}^{R} \int_{0}^{\rho} x(s) d s d \rho x\left(t_{i}\right)+\int_{0}^{R} \int_{\rho}^{t_{i}} x\left(t_{i}+\rho-s\right) x(s) d s d \rho=\int_{0}^{R} f^{\delta}\left(t_{i}+\rho\right) d \rho
$$

for $i=1,2, \ldots, N$. We will use the rectangular quadrature rule to approximate integrals on $[0, R]$, i.e.,

$$
\int_{0}^{R} g(\rho) d \rho \approx \Delta t \sum_{j=0}^{r-1} g\left(t_{j}\right)
$$

and incorporate this approximation directly into (4.2). Looking at each term in (4.2) we then make the following replacements:

$$
\int_{0}^{R} f^{\delta}\left(t_{i}+\rho\right) d \rho \approx \Delta t \sum_{q=0}^{r-1} f^{\delta}\left(t_{i+q}\right)
$$

and letting $x(t)=\sum_{p=1}^{N} c_{p} \chi_{p}(t)$ for $t \in[0,1]$, we have

$$
\begin{aligned}
2 \int_{0}^{R} \int_{0}^{\rho} x(s) d s d \rho x\left(t_{i}\right) & \approx 2 \Delta t\left(\int_{0}^{0} x(s) d s+\int_{0}^{t_{1}} x(s) d s+\ldots+\int_{0}^{t_{r-1}} x(s) d s\right) c_{i} \\
& =2(\Delta t)^{2}\left(\sum_{l=1}^{r-1} \sum_{m=1}^{l} c_{m}\right) c_{i},
\end{aligned}
$$

where we notice that the coefficient of $c_{i}$ involves only $c_{1}, c_{2}, \ldots, c_{r-1}$. The second term of the left-hand side of (4.2) is more complicated, and, depending on the value of $i$ in relation to $r$, we come to different forms for the term. In general, for any fixed $i=1,2, \ldots, N$, we have

$$
\int_{0}^{R} \int_{\rho}^{t_{i}} x\left(t_{i}+\rho-s\right) x(s) d s d \rho \approx \Delta t \sum_{j=0}^{r-1} \int_{t_{j}}^{t_{i}} x\left(t_{i+j}-s\right) x(s) d s .
$$

Further investigation of equation (4.3) leads us to the following cases.

- If $i<r-1$, then

$$
\begin{aligned}
& \int_{0}^{R} \int_{\rho}^{t_{i}} x\left(t_{i}+\rho-s\right) x(s) d s d \rho \\
& \quad \approx(\Delta t)^{2}\left[\sum_{m=1}^{i} \sum_{l=m}^{i} c_{l} c_{i+m-l}-\sum_{m=i+1}^{r-1} \sum_{l=i+1}^{m} c_{l} c_{i+1+m-l}\right],
\end{aligned}
$$

which is nonlinear in $c_{i}$ and involves all values of $c_{1}, c_{2}, \ldots, c_{r-1}$. 
- If $i=r-1$, then

$$
\int_{0}^{R} \int_{\rho}^{t_{i}} x\left(t_{i}+\rho-s\right) x(s) d s d \rho \approx(\Delta t)^{2} \sum_{m=1}^{i} \sum_{l=m}^{i} c_{l} c_{i+m-l},
$$

which is also nonlinear in $c_{i}$ and involves all values of $c_{1}, c_{2}, \ldots, c_{r-1}$.

- If $i \geq r$, then

$$
\int_{0}^{R} \int_{\rho}^{t_{i}} x\left(t_{i}+\rho-s\right) x(s) d s d \rho \approx(\Delta t)^{2} \sum_{m=1}^{r} \sum_{l=m}^{i} c_{l} c_{i+m-l},
$$

With this result, we notice that specifically for $i=r$, (4) is quadratic in $c_{r}$ once $c_{1}, c_{2}, \ldots, c_{r-1}$ are found. Even better, for $i>r,(4)$ is linear in $c_{i}$ once $c_{1}, c_{2}, \ldots, c_{r}$ are found.

Therefore, to determine the values for $c_{i}, i=1,2, \ldots, N$, we solve the following $N$ equations.

- For $i=1,2, \ldots, r-2$, we have the first $r-2$ equations.

$$
\begin{gathered}
\left(2(\Delta t)^{2} \sum_{l=1}^{r-1} \sum_{m=1}^{l} c_{m}\right) c_{i}+(\Delta t)^{2}\left[\sum_{m=1}^{i} \sum_{l=m}^{i} c_{l} c_{i+m-l}-\sum_{m=i+1}^{r-1} \sum_{l=i+1}^{m} c_{l} c_{i+1+m-l}\right] \\
=\Delta t \sum_{q=0}^{r+1} f^{\delta}\left(t_{i+q}\right) .
\end{gathered}
$$

Note that all values of $c_{1}, c_{2}, \ldots, c_{r-1}$ are involved in any of these $r-2$ equations.

- For $i=r-1$, we have the $(r-1)$-th equation.

$$
\left(2(\Delta t)^{2} \sum_{l=1}^{r-1} \sum_{m=1}^{l} c_{m}\right) c_{i}+(\Delta t)^{2} \sum_{m=1}^{i} \sum_{l=m}^{i} c_{l} c_{i+m-l}=\Delta t \sum_{q=0}^{r+1} f^{\delta}\left(t_{i+q}\right) .
$$

Note that all values of $c_{1}, c_{2}, \ldots, c_{r-1}$ are also involved in this $(r-1)$-th equation. Since we now have $r-1$ equations with $r-1$ unknowns, we are ready to solve $c_{1}, c_{2}, \ldots, c_{r-1}$. We would expect the procedure to this point to be computationally intensive since we are basically solving a system of nonlinear equations for $c_{1}, c_{2}, \ldots, c_{r-1}$.

- Once $c_{1}, c_{2}, \ldots, c_{r-1}$ are found, we can solve for $c_{r}, \ldots, c_{N}$ sequentially by

$$
\left(2(\Delta t)^{2} \sum_{l=1}^{r-1} \sum_{m=1}^{l} c_{m}\right) c_{i}+(\Delta t)^{2} \sum_{m=1}^{r} \sum_{l=m}^{i} c_{l} c_{i+m-l}=\Delta t \sum_{q=0}^{r+1} f^{\delta}\left(t_{i+q}\right)
$$

for $i=r, r+1, \ldots, N$. As noted before, we still have to solve a nonlinear equation for $c_{r}$ since the $r$-th equation is quadratic in $c_{r}$. However, once $c_{1}, c_{2}, \ldots, c_{r}$ have already been determined, the remaining $N-r$ equations can be sequentially solved quickly since the $i$ th equation is linear in $c_{i}$ for $i>r$.

Before turning to numerical examples for this discretization, we first examine whether the method can be modified slightly on $[0, R]$ so that the resulting numerical algorithm avoids the difficulties seen above for obtaining $c_{i}$ when $i=1, \ldots, r$. 
5. Alternate Methods on $[0, R]$. As we have just seen, a standard discretization of the regularization method associated with Theorem 3.1 leads to a sequential numerical method for recovering $x(t)$ for $R<t \leq 1$. Unfortunately, for the $x(t)$ values on the interval $0 \leq t \leq R$, we have to solve a non-sequential nonlinear system of equations which can be numerically expensive. This disadvantage motivates us to look for cheaper alternatives to recover $x$ on the interval $0 \leq t \leq R$. In this section, we will propose some alternative methods and give theoretical basis for them.

In what follows we will show that for any function $x_{R}$ sufficiently close to $\bar{x}$ on $[0, R]$, we may find a unique $\hat{x}_{R}^{\delta} \in L_{2}(0,1)$ for which

$$
\hat{x}_{R}^{\delta}(t)=x_{R}(t), \quad t \in[0, R],
$$

and such that $\hat{x}_{R}^{\delta}$ satisfies equation (2.4) on the restricted interval $(R, 1]$. That is, $\hat{x}_{R}^{\delta} \in L_{2}(0,1)$ satisfies

$$
\alpha_{R}(x) x(t)+F_{R}(x)(t)=f_{R}^{\delta}(t), \quad t \in(R, 1] .
$$

Further, we will see that under suitable conditions on the true solution $\bar{x}$ and the choice of $R=R(\delta)$, the function $\hat{x}_{R}^{\delta}$ is a good approximation of $\bar{x}$ for $\delta$ small. The advantage to this new approach is that we are free to find easier ways of determining an approximation $x_{R}$ to $\bar{x}$ on $[0, R]$ than that obtained by solving equation (2.4) on the interval $[0, R]$.

TheOREM 5.1. Assume that $f^{\delta}$ satisfies the $\mathcal{F}$-data condition and let $\tau_{\text {data }}=1 / 2$ in the case of $\mathcal{F}=C[0,1+\bar{R}]$ and let $\tau_{\text {data }}=2 / 5$ in the case of $\mathcal{F}=L_{2}(0,1+\bar{R})$. Assume that the measure $\eta$ is given satisfying (2.8)-(2.9), and that the autoconvolution problem (1.1) has a positive solution $\bar{x} \in W^{2, \infty}[0,1+\bar{R}]$. satisfying

$$
\bar{x}(0)>13 b^{2} e^{2 \sigma}\left\|\bar{x}^{\prime}\right\|_{\infty}
$$

for $\sigma \geq \sigma_{0}$ and $b \geq 2 \bar{\omega} / \underline{\omega}$, where $\bar{\omega}$, $\underline{\omega}$ are given in (2.9) Assume further that $\left\{x_{R}\right\}_{R \in(0, \bar{R}]}$ is any family of $L_{\infty}(0, R)$ functions satisfying

$$
\sup _{t \in[0, R]}\left|x_{R}(t)-\bar{x}(t)\right| \leq \tilde{C} R,
$$

for $\tilde{C}>0$ satisfying

$$
\tilde{C} \leq b\left\|\bar{x}^{\prime}\right\|_{\infty} .
$$

Then there exist constants $k_{1}>0$ and $\hat{C}>0$, all independent of $R$, such that if $\delta=\delta(R)>0$ satisfies

$$
\delta \leq k_{1} R^{1 / \tau_{\text {data }}},
$$

then for all $R>0$ sufficiently small there exists an unique $\hat{x}_{R}^{\delta} \in L_{2}(0,1)$ with $\hat{x}_{R}^{\delta}=$ $x_{R}(t), t \in(0, R]$, with $\hat{x}_{R}^{\delta}$ satisfying equation (2.4) for $t \in(R, 1]$ and for which

$$
\left\|\hat{x}_{R}^{\delta}-\bar{x}\right\|_{\sigma, R}^{2} \leq \hat{C}^{2} R^{2}
$$

where $\|\cdot\|_{\sigma, R}$ is defined from (2.11) using $\mathcal{C} \in\left(1, \frac{13}{12}\right]$. Finally, if $x_{R} \in L_{\infty}(0, R)$ depends continuously on data $f^{\delta} \in \mathcal{F}$, then so does $\hat{x}_{R}^{\delta} \in L_{2}^{\sigma, R}(0,1)$ for all $R>0$ 
sufficiently small.

Proof. To begin, let $\hat{C}<\frac{\bar{x}(0)}{2 e^{2 \sigma} b}$ and define a new ball around $\bar{x}$ by

$$
\hat{\mathcal{B}}_{R}(\bar{x}) \equiv\left\{x \in L_{2}^{\sigma, R}(0,1):\left.x\right|_{[0, R]}=\left.x_{R}\right|_{[0, R]},\|x-\bar{x}\|_{\sigma, R} \leq \hat{C} R\right\},
$$

noting that $\hat{\mathcal{B}}_{R}(\bar{x}) \subseteq \mathcal{B}(\bar{x}, \hat{C} R)$, where $\mathcal{B}(\bar{x}, \hat{C} R)$ was fundamental to the proof of Theorem 3.7.

Defining the new operator $\bar{H}_{R}$ via

$$
\bar{H}_{R}(x)(t)= \begin{cases}x(t) & \text { if } t \in[0, R] \\ H_{R} x(t) & \text { if } t \in(R, 1],\end{cases}
$$

where $H_{R}$ was defined in (3.19), we will use the contraction mapping theorem to show that the operator $\bar{H}_{R}(x)$ has a fixed point in the ball $\hat{\mathcal{B}}_{R}(\bar{x})$. Let $x \in \hat{\mathcal{B}}_{R}(\bar{x})$. Then since $H_{R} x=x=x_{R}$ on $[0, R]$ and

$$
\int_{0}^{R} e^{-2 \sigma t}\left(x_{R}(t)-\bar{x}(t)\right)^{2} d t \leq \tilde{C}^{2} R^{3}
$$

we have

$$
\begin{aligned}
\left\|\bar{H}_{R} x-\bar{x}\right\|_{\sigma, R}^{2} & \leq \tilde{C}^{2} R^{3}\left(\frac{\mathcal{C}^{2}-1}{R}+1\right)+\int_{R}^{1} e^{-2 \sigma t}\left(H_{R} x(t)-\bar{x}(t)\right)^{2} d t \\
& \leq \tilde{C}^{2} R^{2}\left(\mathcal{C}^{2}-(1-R)\right)+\left\|H_{R}(x)-\bar{x}\right\|_{\sigma, R}^{2} \\
& \leq(\tilde{C} \mathcal{C} R)^{2}+\left\|H_{R}(x)-\bar{x}\right\|_{\sigma, R}^{2}
\end{aligned}
$$

for $R>0$ sufficiently small. Using the proof of Theorem 3.7 (in particular, inequality (3.26)), we find that a sufficient condition for $\left\|\bar{H}_{R} x-\bar{x}\right\|_{\sigma, R} \leq \hat{C} R$ is that

$$
\tilde{C} \mathcal{C}+\frac{1}{\bar{x}(0)} k_{1} b \mathcal{C}+\left\|\bar{x}^{\prime}\right\|_{\infty} b \mathcal{C}+\frac{e^{2 \sigma}}{\bar{x}(0)} b \hat{C}^{2}<\hat{C},
$$

so that letting $k_{1}$ be defined by (3.27) and using condition (5.4) on $\tilde{C}$, we find that inequality (5.8) holds if

$$
L(\hat{C}) \equiv \frac{e^{2 \sigma} b}{\bar{x}(0)} \hat{C}^{2}-\hat{C}+3 b\left\|\bar{x}^{\prime}\right\|_{\infty} \mathcal{C}<0 .
$$

In fact, $L$ has two distinct positive real roots $0<\hat{C}_{1}<\hat{C}_{2}$ under assumption (5.2) and $\mathcal{C} \in\left(1, \frac{13}{12}\right]$. Thus any value of $\hat{C}$ in the interval $\left(\hat{C}_{1},\left(\hat{C}_{1}+\hat{C}_{2}\right) / 2\right)$ will give $L(\hat{C})<0$ and also ensure that $\hat{C}<\frac{\bar{x}(0)}{2 e^{2 \sigma} b}$ as prescribed at the beginning of the proof. Thus $\bar{H}_{R}$ takes $\hat{\mathcal{B}}_{R}(\bar{x})$ into itself.

Let $x_{1}, x_{2} \in \hat{\mathcal{B}}_{R}(\bar{x})$. Then

$$
\begin{aligned}
\left\|\bar{H}_{R}\left(x_{1}\right)-\bar{H}_{R}\left(x_{2}\right)\right\|_{\sigma, R}^{2} & =0+\int_{R}^{1} e^{-2 \sigma t}\left(H_{R}\left(x_{1}\right)(t)-H_{R}\left(x_{2}\right)(t)\right)^{2} d t \\
& \leq\left\|H_{R}\left(x_{1}\right)-H_{R}\left(x_{2}\right)\right\|_{\sigma, R}^{2}
\end{aligned}
$$


and thus from the proof of Theorem 3.7 we have that the conditions already placed on $\hat{C}$ are sufficient for $\bar{H}_{R}$ to be a contraction in the ball $\hat{\mathcal{B}}_{R}(\bar{x})$.

Finally, we refine our definition of $\hat{C}$ further to ensure that the ball $\hat{\mathcal{B}}_{R}(\bar{x})$ is nonempty. We note from (5.4) and (5.2) that

$$
\tilde{C} \leq \frac{\bar{x}(0)}{13 b e^{2 \sigma}}=\frac{2}{13} \frac{\hat{C}_{1}+\hat{C}_{2}}{2}
$$

so that if we further restrict $\hat{C}$ to lie in the (nonempty) intersection of the intervals $\left.\left(\frac{2}{13}\left(\hat{C}_{1}+\hat{C}_{2}\right) / 2\right),\left(\hat{C}_{1}+\hat{C}_{2}\right) / 2\right)$ and $\left.\left(\hat{C}_{1},\left(\hat{C}_{1}+\hat{C}_{2}\right) / 2\right)\right)$ we have that

$$
\tilde{C} \leq \hat{C}
$$

Thus if we define $\hat{x}_{R} \in L_{2}^{\sigma, R}(0,1)$ via

$$
\hat{x}_{R}= \begin{cases}x_{R}(t) & \text { if } t \in[0, R] \\ \bar{x}(t) & \text { if } t \in(R, 1],\end{cases}
$$

we have from (5.7) that

$$
\left.\left\|\hat{x}_{R}-\bar{x}\right\|_{\sigma, R}^{2} \leq \hat{C}^{2} R^{2}\left(\mathcal{C}^{2}-1+R\right)\right)+0 \leq \hat{C}^{2} R^{2}
$$

for $R>0$ sufficiently small. Thus $\hat{x}_{R} \in \hat{\mathcal{B}}_{R}(\bar{x})$.

Continuous dependence of $\hat{x}_{R}^{\delta}$ on data $f^{\delta}$ follows arguments similar to those in Theorem 3.7.

As in Corollary 3.8 we may dispense with condition (5.2) provided that we have access to an appropriate approximation $\hat{x}(0 ; R)$ of $\bar{x}(0)$ and that we make use of this information in the construction of our measure $\eta$. See Remark 3.3 for more information on the construction of such a measure.

COROllary 5.2. Assume that $f^{\delta}$ satisfies the $\mathcal{F}$-data condition and let $\tau_{\text {data }}=$ $1 / 2$ in the case of $\mathcal{F}=C[0,1+\bar{R}]$ and let $\tau_{\text {data }}=2 / 5$ in the case of $\mathcal{F}=L_{2}(0,1+\bar{R})$. Let the solution $\bar{x} \in W^{2, \infty}[0,1+\bar{R}]$ of (1.1) be positive. Assume that the measure $\eta$ satisfies (2.8)-(2.9) with $\bar{\omega}, \underline{\omega}$ in (2.9) satisfying (3.4) for all $R>0$ sufficiently small, where $\tilde{K}$ is independent of $\bar{R}$ and $\bar{x}$. Assume further that $\left\{x_{R}\right\}_{R \in(0, \bar{R}]}$ is any family of $L_{\infty}(0,1)$ functions satisfying

$$
\sup _{t \in[0, R]}\left|x_{R}(t)-\bar{x}(t)\right| \leq \tilde{C} R
$$

for some $\tilde{C}>0$.

Then there exist constants $k_{1}>0$ and $\hat{C}>0$, all independent of $R$, such that if $\delta=\delta(R)>0$ satisfies

$$
\delta \leq k_{1} R^{1 / \tau_{\text {data }}}
$$

then there exists an unique $\hat{x}_{R}^{\delta} \in L_{2}(0,1)$ with $\hat{x}_{R}^{\delta}=x_{R}(t), t \in(0, R]$, with $\hat{x}_{R}^{\delta}$ satisfying equation (2.4) for $t \in(R, 1]$ and for which

$$
\left\|\hat{x}_{R}^{\delta}-\bar{x}\right\|_{\sigma, R}^{2} \leq \hat{C}^{2} R^{2}
$$


where $\|\cdot\|_{\sigma, R}$ is defined from (2.11) using $\mathcal{C}=\sqrt{2}$ and $\sigma \geq \sigma_{0}$. Finally, if it is known that $x_{R} \in L_{\infty}(0, R)$ depends continuously on data $f^{\delta} \in \mathcal{F}$, then so does $\hat{x}_{R}^{\delta} \in L_{2}^{\sigma, R}(0,1)$, for all $R>0$ sufficiently small.

Proof. Define $b$ satisfying both $b \geq 2 \bar{\omega} / \underline{\omega}$ and $\tilde{C} \leq b\left\|\bar{x}^{\prime}\right\|_{\infty}$ and let $\tilde{b}=b / \bar{x}(0)$. As in Corollary 3.8 we may assume without loss of generality that the original equation (1.1) has been rescaled so that

$$
\left\|\bar{x}^{\prime}\right\|_{\infty} \bar{x}(0)<\frac{\sqrt{2} e^{-2 \sigma}}{24 \tilde{b}^{2}} .
$$

Then following the proof of Theorem 5.1, a sufficient condition for $\left\|\bar{H}_{R} x-\bar{x}\right\|_{\sigma, R}<\hat{C} R$ is that

$$
L(\hat{C}) \equiv e^{2 \sigma} \tilde{b} \hat{C}^{2}-\hat{C}+3\left\|\bar{x}^{\prime}\right\|_{\infty} \bar{x}(0) \tilde{b} \mathcal{C}<0,
$$

so for $\mathcal{C}=\sqrt{2}$ and under the rescaling in (5.12) we have that $L$ has two distinct positive roots $0<\hat{C}_{1}<\hat{C}_{2}$. The remainder of the proof of Theorem 5.1 then carries over exactly as before, with the exception that we now have from (5.12)

$$
\tilde{C} \leq \tilde{b}\left\|\bar{x}^{\prime}\right\|_{\infty} \bar{x}(0)<\frac{\sqrt{2}}{12} \frac{1}{2 e^{2 \sigma \tilde{b}}}=\frac{\sqrt{2}}{12}\left(\frac{\hat{C}_{1}+\hat{C}_{2}}{2}\right)
$$

so that the arguments in the proof of Theorem 5.1 remain valid if we select $\hat{C}$ in the (nonempty) intersection of the intervals $\left.\left(\frac{\sqrt{2}}{12}\left(\hat{C}_{1}+\hat{C}_{2}\right) / 2\right),\left(\hat{C}_{1}+\hat{C}_{2}\right) / 2\right)$ and $\left.\left(\hat{C}_{1},\left(\hat{C}_{1}+\hat{C}_{2}\right) / 2\right)\right)$.

Remark 5.3. The results of Theorem 5.1 and Corollary 5.2 indicate that, as before, under the a priori conditions on $R=R(\delta)$ that

$$
\left(\delta / k_{1}\right)^{\tau_{\text {data }}} \leq R(\delta) \leq c \delta^{p}
$$

for any $c>0$ and $p \in\left(0, \tau_{\text {data }}\right]\left(c \geq k_{1}^{-\tau_{\text {data }}}\right.$ if $\left.p=\tau_{\text {data }}\right)$ as $\delta \rightarrow 0$, we obtain

$$
\left\|x_{R(\delta)}^{\delta}-\bar{x}\right\|_{L_{2}(0,1)}=\mathcal{O}\left(\delta^{p}\right) \text { as } \delta \rightarrow 0,
$$

with optimal rate obtained for $p=\tau_{\text {data. }}$. Thus the optimal convergence rate for continuous data is $\mathcal{O}\left(\delta^{1 / 2}\right)$ and the optimal convergence rate for $L_{2}$ data is $\mathcal{O}\left(\delta^{2 / 5}\right)$.

As mentioned earlier, Theorem 5.1 and Corollary 5.2 provide us the freedom of finding easier ways of determining an approximation $x_{R}$ to $\bar{x}$ on $[0, R]$ than that obtained by solving the local regularization equation $(2.4)$ on the interval $[0, R]$. One way to find such an $x_{R}$ (and to find an $\mathcal{O}(R)$ approximation $\hat{x}(0 ; R)$ of $\bar{x}(0)$, which is of particular use in Corollaries 3.8 and 5.2 via Remark 3.3) is by a standard discretization of the unregularized problem

$$
G(x)(t)=\int_{0}^{t} x(t-s) x(s) d s=f^{\delta}(t), \quad t \in[0, R],
$$

(with noisy data $f^{\delta}$ ) on the interval $[0, R]$. As is well-known, coarse discretization is a form of regularization; however the ill-posedness of (5.13) can lead to significant 
errors in $x_{R}(t)$ for large $t$, even more so when the discretization stepsize is small. It is for this reason that the standard discretization will only be used on on a small interval of length $R$.

We will describe here one simple discretization of (5.13) which is suitable for use with Theorem 5.1 and Corollary 5.2. Let $K=K(R) \geq 1$ be an integer and partition the interval $[0, R]$ into $K$ equal-length subintervals; i.e., let

$$
t_{i}=i \Delta t, \quad i=0,1, \ldots, K, \quad \Delta t=R / K .
$$

Defining $\chi_{i}(t)$ as in the last section (only now for $i=1,2, \ldots, K$ ), we define $\mathcal{S}_{K}=$ $\operatorname{span}\left\{\chi_{i}, i=1,2, \ldots, K\right\}$ and seek $x_{R} \in \mathcal{S}_{K}$, i.e.,

$$
x_{R}(t)=\sum_{l=1}^{K} c_{l} \chi_{l}(t)
$$

satisfying the collocation equations

$$
G(x)\left(t_{i}\right)=\int_{0}^{t_{i}} x\left(t_{i}-s\right) x(s) d s=f^{\delta}\left(t_{i}\right), \quad i=1,2, \ldots, K,
$$

Therefore,

$$
\sum_{\gamma=1}^{i} \int_{t_{\gamma-1}}^{t_{\gamma}}\left[\sum_{l=1}^{K} c_{l} \chi_{l}\left(t_{i}-s\right)\right]\left[\sum_{p=1}^{K} c_{p} \chi_{p}(s)\right] d s=f^{\delta}\left(t_{i}\right), \quad i=1,2, \ldots, K,
$$

or

$$
\sum_{\gamma=1}^{i} c_{i-\gamma+1} c_{\gamma}=\frac{f^{\delta}\left(t_{i}\right)}{\Delta t}, \quad i=1,2, \ldots, K
$$

The collocation equations (5.16) allow us to explicitly solve for $c_{i}, i=1,2, \ldots, K$, provided that $f^{\delta}\left(t_{1}\right)>0$. That is,

$$
c_{1}=\sqrt{\frac{f^{\delta}\left(t_{1}\right)}{\Delta t}}
$$

and if $c_{1}, \ldots, c_{i-1}$ have been found already, $c_{i}$ is determined by

$$
c_{i}=\frac{\frac{f^{\delta}\left(t_{i}\right)}{\Delta t}-\left(c_{i-1} c_{2}+\ldots+c_{2} c_{i-1}\right)}{2 c_{1}}
$$

therefore, $c_{2}, c_{3}, \ldots, c_{K}$ can be found uniquely, explicitly, and sequentially from (5.18).

The following result is proved using standard techniques (see the details in [8]). If the constant $\tilde{C}$ in (5.21) is not too large (a condition needed for Theorem 5.1 only, not for Corollary 5.2), it follows that the collocation-based approximation $x_{R}$ is suitable for use in methods based on Theorem 5.1 or Corollary 5.2. That is, we may use $x_{R}$ as an approximation for $\bar{x}$ on $[0, R]$ and then use the local regularization equation $(2.4)$ to find an approximation for $\bar{x}$ on $[R, 1]$. We note that the condition $K=K(R) \leq \tilde{M}$ for all $R>0$ sufficiently small is not a serious constraint as we generally expect $K \geq 1$ to be a fixed integer so that the stepsize $\Delta t=R / K$ decreases as $R \rightarrow 0$. However, not 
surprisingly since we are now dealing with a discretization of the original problem, we do need pointwise values of $f^{\delta}$ on $[0, R]$ and we require that they be within $\mathcal{O}\left(R^{2}\right)$ of the corresponding values of $f$ on that interval.

Corollary 5.4. Assume the autoconvolution problem (1.1) has a positive solution $\bar{x} \in C^{1}[0,1]$. Let $x_{R}=\sum_{l=1}^{K} c_{l} \chi_{l}(t)$ be the unique solution of the discrete autoconvolution equation (5.15) on the interval $[0, R]$, where the constants $c_{i}, i=1,2, \ldots, K$, are specified in (5.17) and (5.18) and $K=K(R) \geq 1$ is an integer. Then if there exists a constant $\tilde{M}>0$, such that $K=K(R) \leq \tilde{M}$ uniformly in $R$, and $f^{\delta}$ is piecewise continuous on $[0, R]$ with

$$
\left|f^{\delta}(t)-f(t)\right| \leq \delta \leq k_{1} R^{2}, \quad t \in[0, R],
$$

convergence of $x_{R}(t)$ to the true solution $\bar{x}(t)$ for $t \in[0, R]$ occurs at the collocation points as $R \rightarrow 0$, i.e.,

$$
\left|x_{R}\left(t_{i}\right)-\bar{x}\left(t_{i}\right)\right| \sim \mathcal{O}(R), \quad \text { for } i=1,2, \ldots, K,
$$

for $R$ sufficiently small. Further, we have a constant $\tilde{C}$ depending on $\bar{x}$ but independent of $R$ such that

$$
\left|x_{R}(t)-\bar{x}(t)\right| \leq \tilde{C} R
$$

for $R$ sufficiently small and all $t \in[0, R]$.

REMARK 5.5. It is worth noting that the results of Theorem 5.1 are improved if we require that $x_{R}$ be an $\mathcal{O}\left(R^{p}\right)$ approximation to $\bar{x}$ on $[0, R]$ for some $p>1$ [8]. This result is of use, for example, if we know both $\bar{x}(0)$ and $\bar{x}^{\prime}(0)$ so that we form an $\mathcal{O}\left(R^{2}\right)$ approximation of $\bar{x}$ on $[0, R]$ via $x_{R}(t)=\bar{x}(0)+\bar{x}^{\prime}(0) t$. However, as can be seen in [8], such approximations on $[0, R]$ actually perform worse (locally) in numerical examples than do approximations based on the simple $\mathcal{O}(R)$ collocation-based discretization of (5.13) given above. The reason for this is likely due to the fact that the approximation based on a Taylor expansion does not make good use of all of the available data $f^{\delta}$ on $[0, R]$, as does the collocation-based discretization.

5.1. Operation Count for the Alternative Method. If we let $r=K$ in (5.17)-(5.18), then the cost of computing $c_{1}, c_{2}, \ldots, c_{r}$ from $(5.17)-(5.18)$ is $\mathcal{O}\left(r^{2} / 2\right)$ flops. The remaining steps in the alternative approach require the sequential solution of (4.4) for $c_{r+1}, \ldots c_{N}$. We can rewrite equation (4.4) for $c_{i}$ as

$$
\left(D_{1}+D_{2}\right) c_{i}+D_{3}(i)=D_{4}(i), \quad i=r+1, \ldots, N,
$$

where $D_{j}, j=1, \ldots, 4$ are constants which independent of $i$ in the case of $D_{1}$ and $D_{2}$,

$$
\begin{aligned}
D_{1} & =\left(2 \sum_{l=1}^{r-1} \sum_{m=1}^{l} c_{m}\right), \quad D_{2}=2 \sum_{m=1}^{r} c_{m} \\
D_{3}(i) & =\sum_{m=1}^{r} \sum_{l=m+1}^{i-1} c_{l} c_{i+m-l}, \quad D_{4}(i)=\frac{1}{\Delta t} \sum_{q=0}^{r+1} f^{\delta}\left(t_{i+q}\right) .
\end{aligned}
$$


The computation of $D_{1}$ and $D_{2}$ require one-times costs of order $\mathcal{O}(r)$ flops each, while the cost of $D_{3}(i)$ and $D_{4}(i)$ are $\mathcal{O}\left(2 i r-r^{2}-3 r\right)$ and $\mathcal{O}(r)$ flops respectively, computations which are done for $i=r+1$ to $i=N$. Thus the total cost of the alternative method described in this section (assuming $r \ll N)$ is $\mathcal{O}\left(r N^{2}-r^{2} N\right)$.

Operation counts for the Lavrent'ev method are likely to be similar to those found above (i.e., $\mathcal{O}\left(c N^{2}\right)$ for some constant $c>0$ ), however fairly accurate knowledge of the value of $\bar{x}(0)$ is of importance if one is to make use of this method; in addition, one requires an initial guess $x^{\star}$ for implementation of Lavrent'ev. For comparison with methods which do not preserve the causal nature of the nonlinear autoconvolution problem, we have been unable to find operation counts for Tikhonov regularization applied to this nonlinear problem (and of course there are many different approaches - methods using Newton or Levenberg-Marquardt, descent methods such as steepest descent, etc. - that one might take to solve the nonlinear Tikhonov problem). Without a suitable comparison, we'll simply note that cost for a direct implementation of Tikhonov regularization on a general linear problem is $\mathcal{O}\left(N^{3}\right)$ flops [10]. Some actual computational costs (in seconds) are provided in Examples 3 and 4 below.

6. Numerical Results. The examples in this section provide evidence of the effectiveness of the local regularization methods applied to the autoconvolution equation. The local regularization methods have advantages over existing methods in that they do not require an initial guess $x^{\star}$ and they maintain the causal nature of the problem at least for the majority of the domain, leading to very rapid solution methods. One drawback, of course, is that the theory requires that true solutions $\bar{x}$ should not cross the $t$-axis. However, as Examples 3 and 4 below illustrate, the method appears to perform perfectly well in this situation.

Henceforth we will refer to Method 1 as the collocation-based discretization derived in Section 4 for the local regularization equation (2.4), i.e., a discretization of the continuous local regularization method which is rigorously justified by Theorem 3.7. Recall that Method 1 which requires solving non-sequential nonlinear equations for $c_{1}, \ldots, c_{r}$, and sequential linear equations for $c_{r+1}, \ldots, c_{N}$.

In contrast, Method 2 will refer to the method of determining $c_{1}, \ldots, c_{r}$ via (5.17)-(5.18) (where we set $K=r$ ) and $c_{r+1}, \ldots, c_{N}$ via (4.4). In Method 2, all coefficients are found using a procedure that's fully sequential and linear in the unknown coefficient at each step. This algorithm is a discretization of the continuous local regularization method which is rigorously justified by Theorem 5.1. It is interesting to note in our numerical examples below that that the fully sequential linear Method 2, which is significantly easier to implement and considerably faster in terms of computational times, actually performs as well as the (slower) partly-nonlinear Method 1.

In order for easy comparison with numerical tests in the literature for existing methods (a Tikhonov regularization approach using total variation constraints in [13] and a Lavrent'ev approach in [16]), we demonstrate the recovery of both a continuous $\bar{x}$ and a discontinuous $\bar{x}$ using Methods 1 and 2 above. We select our true solution $\bar{x}$ ahead of time, then generate the data function $f$ by integration, $f(t)=\int_{0}^{t} \bar{x}(t-$ $s) \bar{x}(s) d s$, for $t \in[0,1]$. The perturbed data $f^{\delta}$ was then produced by adding uniformly distributed noise from the interval $[-\delta f(t), \delta f(t)]$ to the discrete values of $f(t)$ for $t=t_{i}$, where $i=1,2, \ldots, N$. Each example within the same noise-level $\delta$ was found using the same sampled data $f^{\delta}$.

In the figures below we display the recovered solution against the true solution $\bar{x}$, first without any special regularization (except for that due to discretization alone), 
and then with regularization using Methods 1 and 2. In all figures presented below, the true solution $\bar{x}$ is plotted as a dashed line, while the solid line expresses the approximate solution computed according to the specified method. The regularized solutions end before reaching $t=1$ in Examples 1 and 2 because we have chosen not to extend our data past the interval $[0,1]$ in those examples; to have regularized solutions on all of $[0,1]$ one needs to make use of data $f^{\delta}$ on $[0,1+R]$.

6.1. Example 1: Continuous true solution $\bar{x}$. The true solution in this example is a continuous function $\bar{x}(t)=1-3(t-1 / 2)^{2}, 0 \leq t \leq 1$, with the true data then given by $f(t)=\frac{3}{10} t^{5}-\frac{3}{2} t^{4}+t^{3}+\frac{3}{4} t^{2}+\frac{1}{16} t$, for $0 \leq t \leq 1$. For Figures $6.1-6.3$, we use relative noise level $\delta=10^{-3}, N=100$ and $r=4$ for the regularized problems. In Figure 6.1, we show the unregularized numerical solution while in Figures $6.2-6.3$ we show the regularized solution using Methods 1 and 2 as introduced in the last section.

For Figures 6.4-6.6, we repeat the above example using a larger relative noise level $\delta=10^{-2}, N=100$ and using $r=4$ for Method 1 and $r=6$ for Method 2. (We selected the value of $r$, small, which gave the best results in each case visually.)

6.2. Example 2: Discontinuous true solution $\bar{x}$. In this example, we will use a step function as our true solution; $\bar{x}$ and true data $f$ are given by

$$
\bar{x}(t)=\left\{\begin{array}{ll}
0.5 & \text { if } t \in[0,0.5], \\
0.25 & \text { if } t \in(0.5,0.8], \\
0.75 & \text { if } t \in(0.8,1],
\end{array} \quad f(t)= \begin{cases}0.25 t & \text { if } t \in[0,0.5] \\
0.125 & \text { if } t \in(0.5,0.8] \\
0.5 t-0.275 & \text { if } t \in(0.8,1]\end{cases}\right.
$$

For all the figures shown in this example, we use relative noise level $\delta=10^{-3}, N=200$ and $r=4$ for the regularized problem. In Figure 6.7, we show the solution without any special regularization, while Figures 6.8-6.9 show the regularized solution using Methods 1 and 2. The results for both methods are much better than was presented in $[13,16]$. It is worth noting that the true solution $\bar{x}$ here does not satisfy the assumptions of Theorem 3.7 (which are only sufficient conditions for convergence anyway).

For comparison with local regularization, we show in Figure 6.10 the results of Tikhonov regularization and Lavrent'ev regularization applied to this same problem. In each case, regularization parameters were selected as those providing the best visual fit between approximate and true solutions. For our implementation of Lavrent'ev, the true value of $\bar{x}(0)$ was incorporated into the approximating equation, and the regularization parameter $\alpha=.01555$ was used. For Tikhonov regularization, we used the parameter $\alpha=10^{-22}$ with starting guess of $x(t)=\sqrt{f^{\delta}\left(t_{1}\right) / \Delta t}$; for more information about our implementation of the nonlinear Tikhonov scheme, refer to Example 3 below.

6.3. Example 3: Nonpositive true solution $\bar{x}$. Although the theory developed here requires that $\bar{x}$ be strictly positive (resp. strictly negative), in practice the numerical methods developed in this paper can still be applied to situations where the unknown $\bar{x}$ crosses the $t$-axis. We illustrate with an example where the true solution is given by $\bar{x}(t)=\sin 8 t+(1 / 4), 0 \leq t \leq 1$, and the associated true data is $f(t)=\frac{5}{80}(1+t-\cos 8 t+\sin 8 t)-\frac{1}{2} t \cos 8 t, 0 \leq t \leq 1$. We use a relative noise level $\delta=.01$ and $N=100$, and in Figures $6.11-6.12$ show the results of no regularization as well as those for Tikhonov regularization, Lavrent'ev regularization, and local regularization, all applied to the same data $f^{\delta}$.

In the case of local regularization we use Method 2 and $r=7$, and in so doing make use of data on the extended interval [0,1.07]; for a fair comparison, we also 


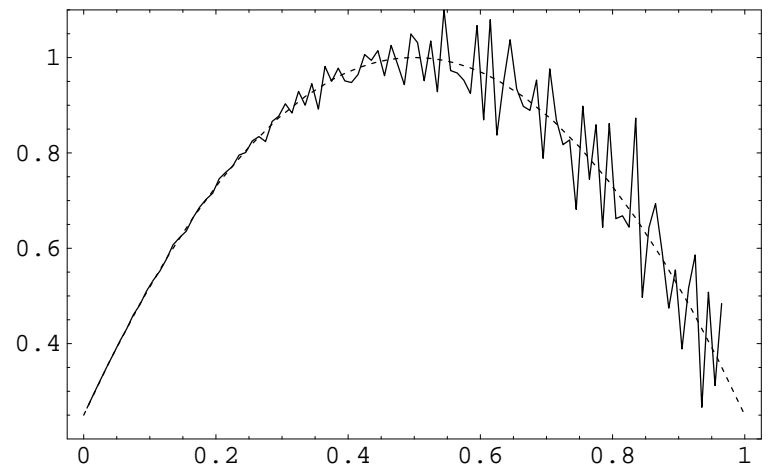

FIG. 6.1. Example 1. Solution without regularization, $\delta=10^{-3}, N=100$

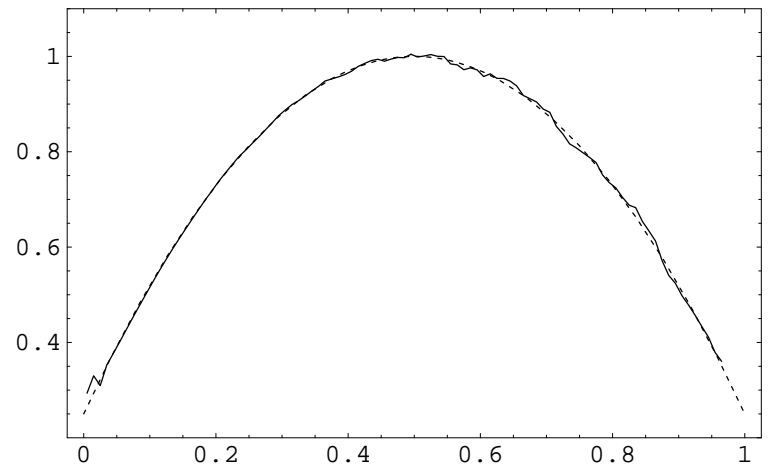

FIG. 6.2. Example 1. Solution obtained by Method 1, $\delta=10^{-3}, N=100, r=4$

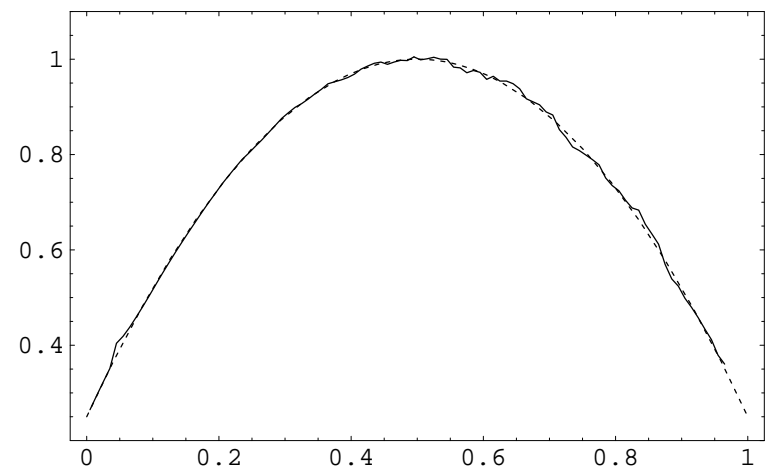

FIG. 6.3. Example 1. Solution obtained by Method 2, $\delta=10^{-3}, N=100, r=4$ 


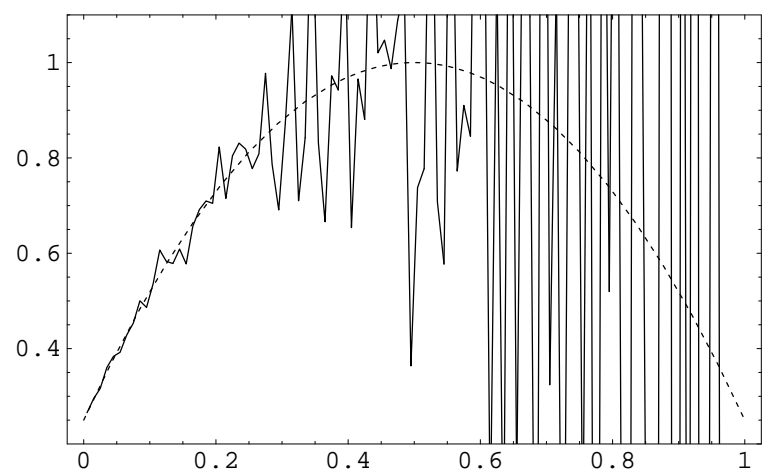

FIG. 6.4. Example 1. Solution without regularization, $\delta=10^{-2}, N=100$

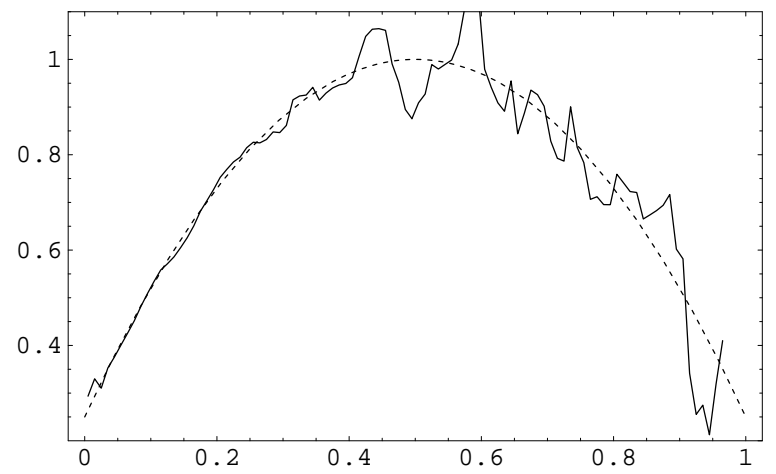

FIG. 6.5. Example 1. Solution obtained by Method 1, $\delta=10^{-2}, N=100, r=4$

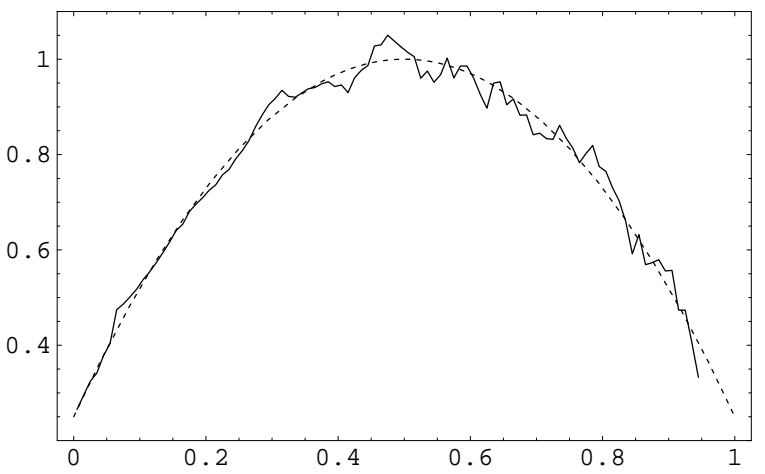

FIG. 6.6. Example 1. Solution obtained by Method 2, $\delta=10^{-2}, N=100, r=6$ 


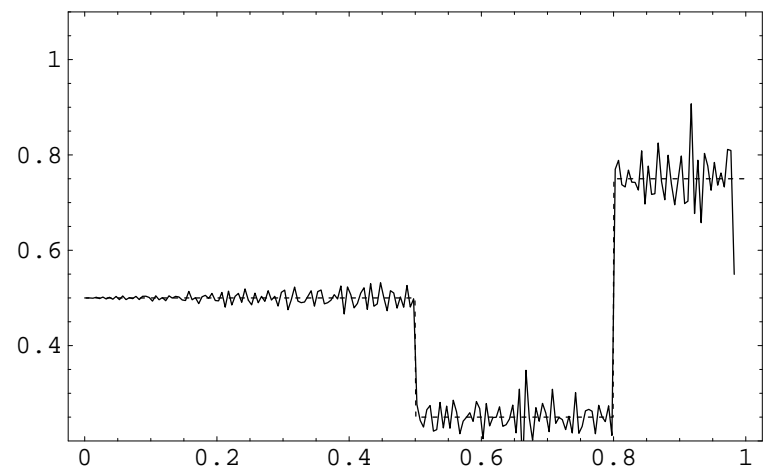

FIG. 6.7. Example 2. Solution without regularization, $\delta=10^{-3}, N=200$

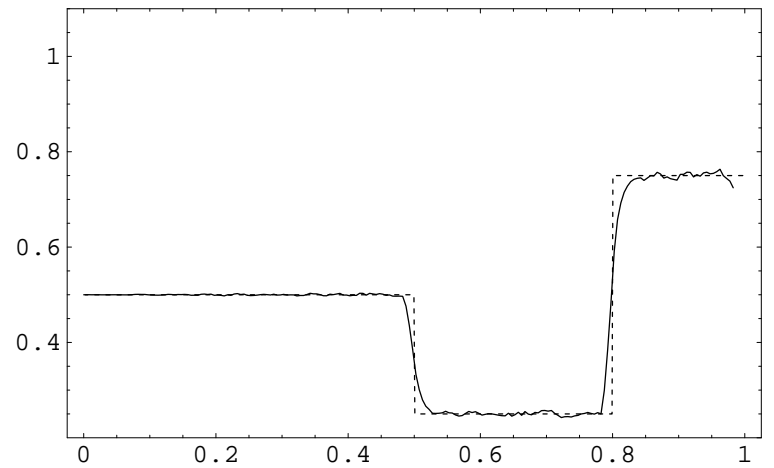

FIG. 6.8. Example 2. Solution obtained by Method 1, $\delta=10^{-3}, N=200, r=4$

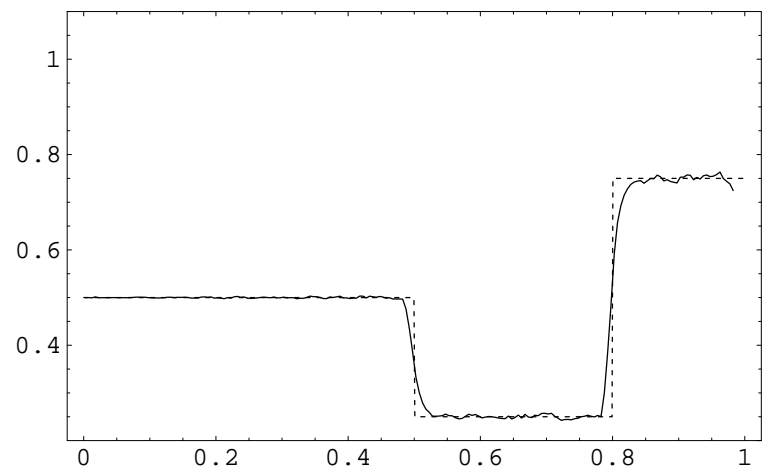

FIG. 6.9. Example 2. Solution obtained by Method 2, $\delta=10^{-3}, N=200, r=4$ 

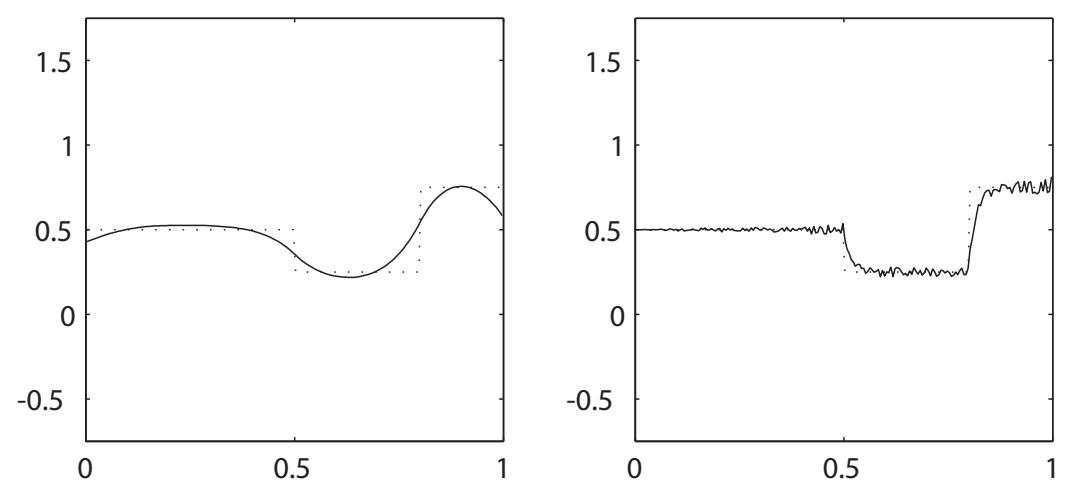

FIG. 6.10. Example 2, continued. Left: Tikhonov solution. Right: Lavrent'ev solution.

allow Tikhonov regularization to make use of the same extended interval for its computations; however, only the results on $[0,1]$ are displayed for both methods. We note that the Lavrent'ev method is solved sequentially without the use of future data, so additional data past the interval $[0,1]$ does not change its result. For the Tikhonov and Lavrent'ev methods we use the regularization parameters $\alpha=10^{-6}$ and $\alpha=0.4$, respectively, incorporating the true value of $\bar{x}(0)$ into the Lavrent'ev approximating equation. Minimization of the nonlinear Tikhonov regularization functional (with regularization term $\alpha\|x\|^{2}$ ) is accomplished in these examples using the Matlab function fminunc with initial guess provided by the constant function $x(t)=c_{1}$, where $c_{1}$ is the first value of the approximation found in Method 2 for local regularization, i.e., $c_{1}=\sqrt{f^{\delta}\left(t_{1}\right) / \Delta t}$. The regularization parameters used for all methods are the best ones found by visual comparison with the true solution.

We note that Lavrent'ev provides a shifted approximation; decreasing $\alpha$ does reduce the shift, but at the same time causes large distortions in the shape of the solution. The fact that Tikhonov method gives a smoother solution than local regularization can be expected due to the global vs local smoothing associated with the two methods. The computing time for Lavrent'ev and local regularization is about the same as that for the unregularized solution $(0.01 \mathrm{sec}$ on a $2.21 \mathrm{GHz}$ dual core machine), while Tikhonov regularization requires 166 times that much computing time $(1.66 \mathrm{sec})$.

6.4. Example 4: Nonpositive true solution $\bar{x}$. In this example we take the true $\bar{x}$ from Example 1 above and shift it down so that it crosses the $t$-axis in two places. That is, we use $\bar{x}(t)=(1 / 2)-3(t-1 / 2)^{2}$ for $0 \leq t \leq 1$, with true data $f(t)=\frac{3}{10} t^{5}-\frac{3}{2} t^{4}+2 t^{3}-\frac{3}{4} t^{2}+\frac{1}{16} t$, for $0 \leq t \leq 1$. In this example we use a relative noise level $\delta=.015$ and $N=200$, and in Figures 6.15-6.16 we again show the results of no regularization, Tikhonov regularization, Lavrent'ev regularization, and local regularization, for the same data $f^{\delta}$.

The true data $f$ is graphed in Figure 6.14. The difference between the data $f$ in Example 3 (Figure 6.13) and the data $f$ in Figure 6.14 for this example is important in understanding the difference between the way Tikhonov regularization and local regularization perform in this particular case. Note that here the true data $f$ is nearly zero for the first third to half of the interval. This means that with even small amounts of added error, the signal-to-noise ratio is likely to be so small as to make the solution unrecoverable if only the data from the first third of the interval 

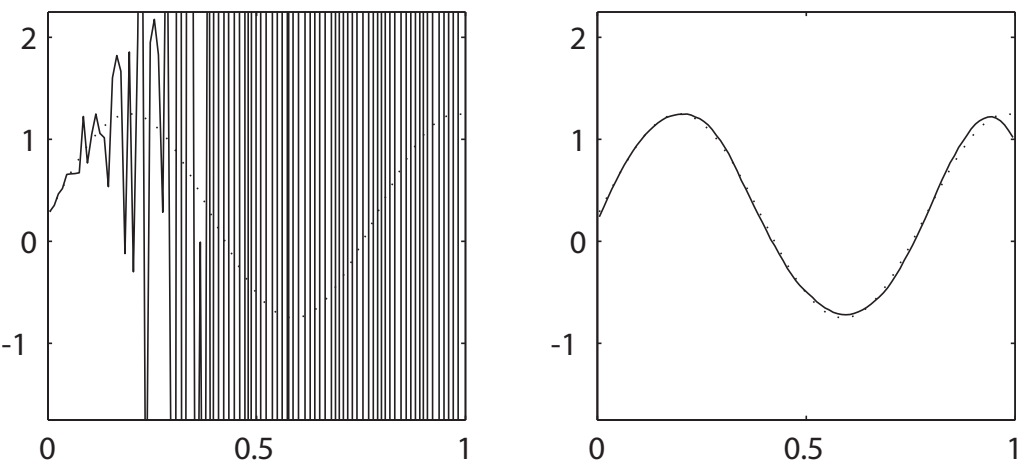

FIG. 6.11. Example 3 with nonpositive true solution, $\delta=10^{-2}$ and $N=100$. Left: unregularized solution (computing time: $0.01 \mathrm{sec}$ ). Right: Tikhonov solution $(1.66 \mathrm{sec})$.
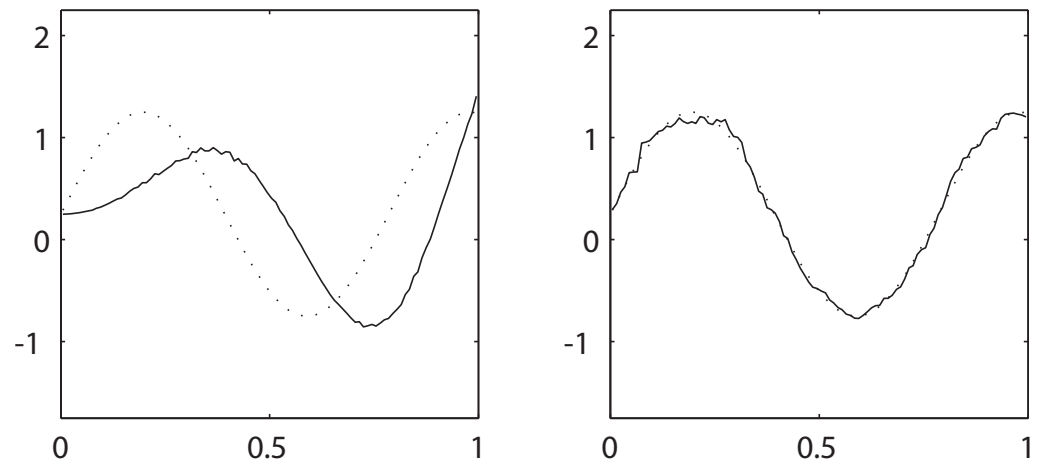

FIG. 6.12. Example 3, continued. Left: Lavrent'ev method (computing time $0.01 \mathrm{sec}$ ). Right: Local regularization (Method 2) with $r=7$ (0.01 sec).

is used. Now Tikhonov regularization uses all the data to compute its regularized approximation, while local regularization constructs a sequential method making use of future data only on the interval $[t, t+r \Delta t]$ to compute the value of the approximate solution at $t$. Thus $r$ will need to be large enough to allow for meaningful information about the data to be obtained in the case of local regularization. In this case, a value of $r=65$ (nearly $1 / 3$ of the interval) is used in order to get good results with local regularization. In contrast, the data in Example 3 does not exhibit this same difficulty with small signal-to-noise data ratios over large regions, so a small value of $r(r=7)$ is acceptable for that example. Fortunately, the need for large $r$ can be determined by a simple inspection of the given (perturbed) data $f^{\delta}$ prior to use of the local regularization method.

Note further that the Lavrent'ev approximation, also found sequentially, uses no future data information in order to compute the value of the approximation at the current time $t$. Thus Lavrent'ev can be expected to fail badly in situations where the signal-to-noise ratio is small over large regions of the domain.

As in Example 3, we allow Tikhonov regularization to make use of the same extended data set as that for local regularization, i.e., of data on the interval $[0,1.325]$. 


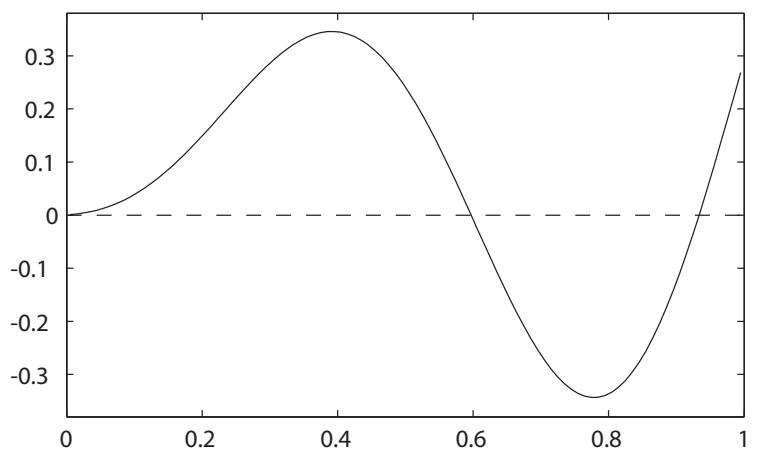

FIG. 6.13. Example 3. True data f.

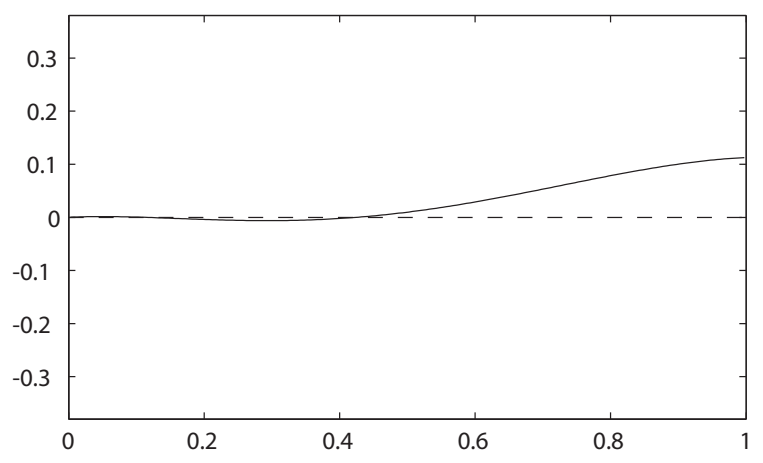

FIG. 6.14. Example 4. True data $f$.

Although the starting value for Tikhonov is the constant function $x(t)=c_{1}$, where now $c_{1}=-\sqrt{f^{\delta}\left(t_{1}\right) / \Delta t}$ ( since $\bar{x}(0)<0$ in this case), Tikhonov regularization actually finds the negative solution $-\bar{x}$ for this problem. (Recall that there are two solutions, $\bar{x}$ and $-\bar{x}$ for every autoconvolution problem; by specifying a positive or negative starting value for each method, we are giving some indication of which of the two solutions to find.)

As expected from the data, Lavrent'ev performs quite poorly in this example. It is possible that the approximation is that for the negative solution of the autoconvolution equation, although the results aren't very convincing. On the other hand, local regularization performs quite well provided $r$ is large enough to get a meaningful local sample of the data. We note that for $r \leq 58$, the local regularization approximation blows up at about $t=1 / 3$, while acceptable results are found for a large range of values of $r \geq 59$. Thus, a simple inspection of the noisy data is very important in order to obtain information about the smallest of $r$ which is likely to gain any meaningful information from data on an interval of length $[t, t+r \Delta t]$.

It is worth observing that, with the large value of $r$ used in this example $(r=$ $65)$, the computing time $(.03 \mathrm{sec})$ for local regularization becomes about 10 times that for the unregularized problem and for Lavrent'ev regularization (.003 sec each); on the other hand, the computing time for Tikhonov regularization (at $5.43 \mathrm{sec}$ ) is 

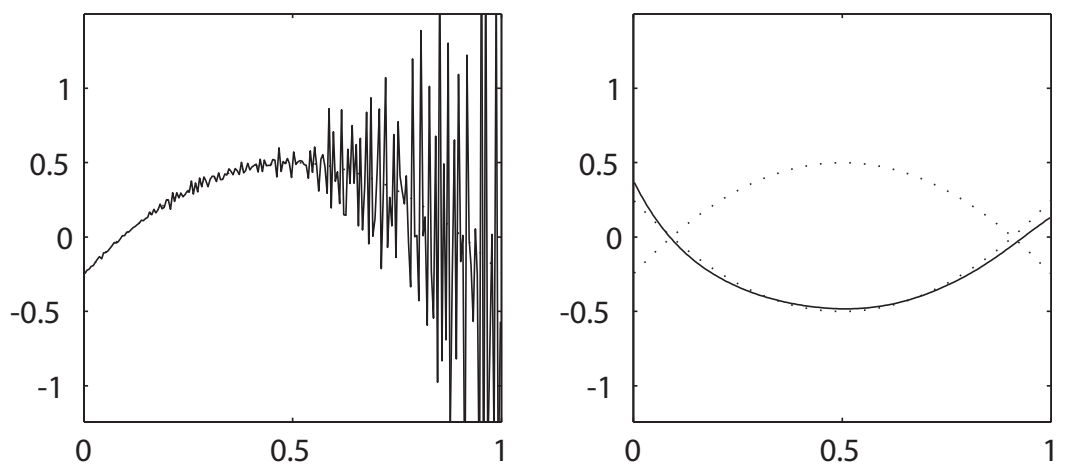

FIG. 6.15. Example 4 with nonpositive true solution, $\delta=1.5 \times 10^{-2}$ and $N=200$. Left: unregularized solution (computing time: $0.003 \mathrm{sec}$ ). Right: Tikhonov solution $(5.43 \mathrm{sec})$ finds an approximation of the negative solution $-\bar{x}$ (both positive and negative true solutions are graphed with dotted lines above).
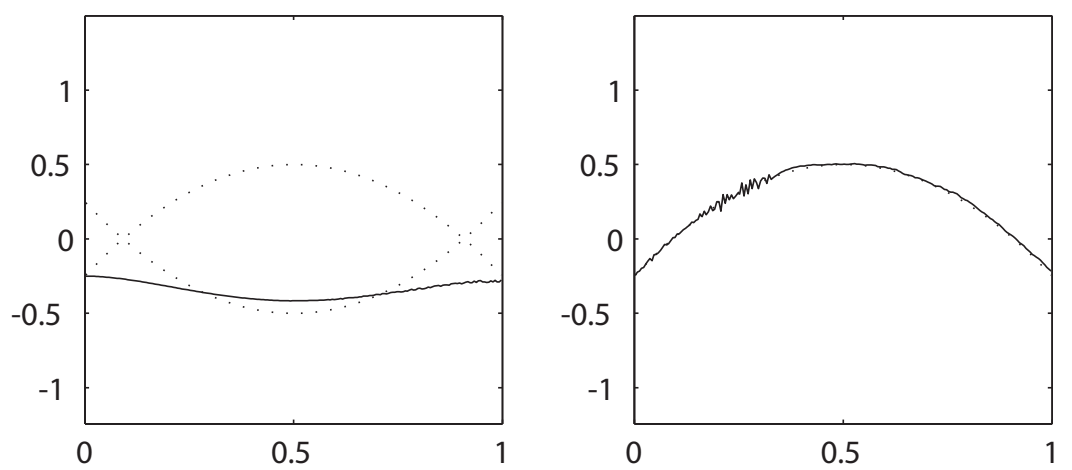

FIG. 6.16. Example 4, continued. Left: Lavrent'ev method (computing time $0.003 \mathrm{sec}$ ). Right: Local regularization (Method 2) with $r=65(0.03 \mathrm{sec})$.

about 180 times that of local regularization. Such differences in computational times become important if discrepancy principles are to be used in the determination of the correct regularization parameters, since such methods require repeated solution of the approximation for different choices of the parameters.

7. Selection of the Regularization Parameter. One of the most commonly asked questions with regard to the local regularization methods is how one picks the regularization parameter $r$ (or $R$ in the continuous version of the regularized equation). This question has been answered for the linear convolution problem with the development of a modified discrepancy principle [3, 4], but remains an open problem (other than the need for minimal values of $r$ in some cases, as discussed in Example 4 above) for nonlinear problems, including the nonlinear autoconvolution equation under study here.

The discrepancy principle is one of the most successful criteria in determining the regularization parameter $\alpha$ for the method of Tikhonov regularization. To summarize the discrepancy principle on the Hilbert space $F$, we assume that the perturbed data 
$f^{\delta}$ has an absolute noise level $\hat{\delta}$, i.e., $\left\|f^{\delta}-f\right\|_{F} \leq \hat{\delta}$. The Tikhonov theory states that for every choice of $\alpha>0$, the unique solution $u_{\alpha}^{\delta}$ of the Tikhonov problem (see, e.g., [10]) associated with the perturbed data $f^{\delta}$ is such that the discrepancy

$$
\delta_{d} \equiv\left\|\mathcal{A} u_{\alpha}^{\delta}-f^{\delta}\right\|_{F}
$$

is monotone in $\alpha$; here $\mathcal{A}$ denotes a linear integral operator. The discrepancy principle is then used to pick $\alpha$ so

$$
\delta_{d}=\tau \hat{\delta}
$$

with $\tau \geq 1$ some constant. In practice, $\tau$ is often picked as $\sqrt{2}$. Development of the discrepancy principle for Tikhonov regularization of nonlinear operator equations can be found in $[30,32]$.

In the following, we use the discrepancy principle to select an "optimal" $r$ for a given relative noise level $\delta$ and fixed discretization parameter $N$. Method 2 is used for all numerical experiments that follow. For local regularization the discrepancy $\delta_{d}$ is not exactly a monotone increasing function of the regularization parameter $r$. Therefore, we modified the principle to select the smallest $r>0$ for which the discrepancy $\delta_{d}$ exceeds the absolute data noise $\hat{\delta}$.

\begin{tabular}{r|c|c}
$r$ & $\hat{\delta}$ & $\delta_{d}$ \\
\hline 4 & .00270788 & .00265035 \\
$\bullet 5$ & .00269173 & .00371632 \\
6 & .00262241 & .00508314 \\
7 & .00261181 & .00663777 \\
8 & .00249610 & .00821011
\end{tabular}

\begin{tabular}{c|c|c}
$r$ & $\hat{\delta}$ & $\delta_{d}$ \\
\hline 8 & .0249610 & .0188627 \\
9 & .0236252 & .0182476 \\
10 & .0231554 & .0194804 \\
11 & .0226741 & .0197377 \\
12 & .0226101 & .0211548 \\
$\bullet 13$ & .0219453 & .0228909 \\
14 & .0219144 & .0182378
\end{tabular}

TABLE 7.1

Example 1 a with $\delta=10^{-3}$ and $\delta=10^{-2}$, respectively.

7.1. Example 1a: A repeat of Example 1, now using the discrepancy principle to select $r$. In Table 7.1 we illustrate how the modified discrepancy principle can be used to determine $r$ for the two numerical tests (associated with the two different noise levels, $\delta=10^{-3}$ and $\delta=10^{-2}$ ) from Example 1 in the last section. We see that $r=5$ is selected for the case of $\delta=10^{-3}$ while $r=13$ is selected for the case of $\delta=10^{-2}$. As expected, the suggested value of $r$ increases as the noise level in the data increases. We show in Figure 7.1 the outcome when using the predicted $r=13$ at noise level of $\delta=.01$; indeed, in comparing with Figure 6.6 (where $r=6$ was used), the relative root-mean-square (rms) error of the reconstructed $x$ is 0.0258251 with $r=13$, which is just about half of the rms error (0.0502236) using $r=6$, when the same set of noisy data is used. We note also that the predicted $r=13$ gives $R=1.3 \times 10^{-1}$, a value which might be seen as more in line with the suggestion made by Theorem 3.1 than the values of $R$ used in Example 1 .

7.2. Example 2a: A repeat of Example 2, now using the discrepancy principle to select $r$. We show in Table 7.2 the results of applying the modified 


\begin{tabular}{r|c|c}
$r$ & $\hat{\delta}$ & $\delta_{d}$ \\
\hline 2 & 0.00133155 & 0.00113062 \\
- 3 & 0.0013285 & 0.00202835 \\
4 & 0.00131227 & 0.00308485
\end{tabular}

TABLE 7.2

Example 2a with relative noise level $\delta=10^{-3}, N=200$ and discontinuous $\bar{x}$

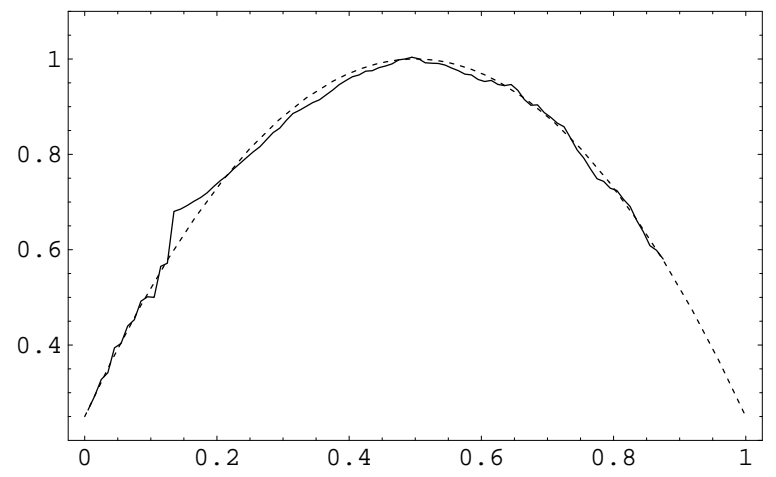

FIG. 7.1. Example 1a. Solution obtained for Example 1 using Method 2 with $\delta=10^{-2} N=100$, $r=13$. For comparison, see Figure 6.6.

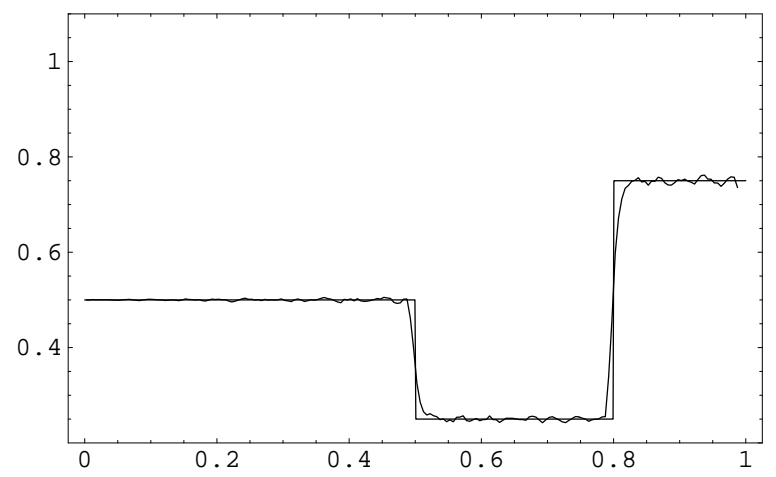

FIG. 7.2. Example 2a. Solution obtained for Example 2 using Method 2 with $\delta=10^{-3}$, $N=200, r=3$. For comparison, see Figure 6.9.

discrepancy principle to Example 2 of the last section; here the value $r=3$ is selected by the principle and the associated numerical solution is shown in Figure 7.2. While Figure 7.2 looks quite similar to Figure 6.9 (where $r=4$ was used), we can quantitatively conclude $r=3$ is a better choice since the relative rms error is 0.072 , which is slightly better than the rms error (0.086) in the case of $r=4$ when the same set of noisy data is used.

Other examples of the use of the modified discrepancy principle for the local regularization of the autoconvolution problem can be found in [8].

8. Conclusion. In this paper we have developed a local regularization theory for the autoconvolution equation, an ill-posed nonlinear Volterra problem. Several local 
regularization methods have been established, all of which provide stable solutions for the autoconvolution problem. The methods also preserve the causal nature of the autoconvolution equation, allowing for numerically fast sequential solution.

Using the underlying idea of local regularization, we formulated the local regularized equation for the autoconvolution problem and proved the convergence of the solution produced by this regularized equation to the true solution of the autoconvolution equation as the noise level in the data shrinks to zero. The convergence rate of $\mathcal{O}\left(\delta^{2 / 5}\right)$ that we obtain for $L_{2}$ data is not as good as that $\mathcal{O}\left(\delta^{1 / 2}\right)$ obtained by Tikhonov regularization and Lavrent'ev regularization. Only with continuous data are we able to reach the $\mathcal{O}\left(\delta^{1 / 2}\right)$ rate of these methods. However, in contrast to these other methods, the theory for local regularization does not rely on an a priori guess for the value of the true solution or of the solution's value at $t=0$, although a source condition does require a particular relationship between $\bar{x}(0)$ and $\left\|\bar{x}^{\prime}\right\|_{\infty}$. The theory for both the local regularization and the Lavrent'ev methods requires a positive true solution $\bar{x}$, a condition not needed for Tikhonov regularization. However, as was shown in Examples 3 and 4, local regularization still performs quite well in numerical testing with nonpositive $\bar{x}$ provided a simple inspection of the noisy data has been made in order to determine a minimal suitable value of the local regularization parameter.

We have also presented two discretization algorithms which illustrate the theory developed in this paper; one algorithm involves the solution of non-sequential nonlinear equations on $[0, R]$; the second algorithm is an improvement in both speed and ease of implementation in that the approximate solution can be determined in a (linear) sequential manner for the entire interval $[0,1]$. The cost for the second algorithm is $\mathcal{O}\left(r N^{2}-r^{2} N\right)$ where $r \ll N$, so that it is of the order of the cost of solving the autoconvolution problem without any regularization whatsoever.

Finally, we have shown numerical results which provide evidence that the local regularization methods developed in this work compare favorably to the other numerical methods for the autoconvolution equation in the literature $[13,16]$, especially in capturing sharp features in the solution. In fact, the numerical results confirm the effectiveness of local regularization methods even in cases not completely falling under the assumptions of the general theory we developed here. Numerical examples also seem to confirm the feasibility of a modified discrepancy principle for the selection of the regularization parameter $r$. The theoretical analysis of this principle is currently under study.

9. Acknowledgment. We wish to thank the referees for helpful comments which improved the exposition of this paper.

\section{REFERENCES}

[1] J. Baumeister, Deconvolution of appearance potential spectra, In: R.Kleinmann, et al.(eds): Direct and inverse boundary value problems. Proc. Conf. Oberwolfach. Lang, Frankfurt am Main (1991) 1-13.

[2] J. V. Beck, B. Blackwell, and C. R. St. Clair, Jr., Inverse Heat Conduction, Wiley-Interscience, $(1985)$.

[3] C. D. Brooks, A discrepancy principle for parameter selection in the local regularization of linear Volterra inverse problems, Ph.D. thesis, Department of Mathematics, Michigan State University (2007).

[4] C. D. Brooks and P. K. Lamm, A discrepancy principle for parameter selection in the local regularization of linear Volterra inverse problems. Preprint (2007). 
[5] A. C. Cinzori, Continuous future polynomial regularization of 1-smoothing Volterra problems, Inverse Problems 20 (2004) 1791-1806.

[6] A. C. Cinzori and P. K. Lamm, Future polynomial regularization of ill-posed Volterra equations, SIAM J.Numer.Anal 37 (2000) 949-979.

[7] C. Cui, Local regularization methods for n-dimensional first-kind integral equations, Ph.D. Thesis, Michigan State University, 2005

[8] Z. Dai, Local regularization for the autoconvolution problem, Ph.D. thesis, Department of Mathematics, Michigan State University (2005).

[9] H. W. Engl, K. Kunisch, A. Neubauer, Convergence rates for Tikhonov regularization of nonlinear ill-posed problems, Inverse Problems 5 (1989) 524-540.

[10] H. W. Engl, M. Hanke, and A. Neubauer, Regularization of Inverse Problems, Kluwer Academic Publishers, Dordrecht, The Netherlands, 1996.

[11] G. Fleischer, B. Hofmann, On inversion rates for the autoconvolution equation, Inverse Problems 12 (1996) 419-435.

[12] G. Fleischer, B. Hofmann, The local degree of ill-posedness and the autoconvolution equation, Nonlinear Analysis, Theory, Methods \& Application. Vol.30, No.6, (1997) 3323-3332.

[13] G. Fleischer, R. Gorenflo, B. Hofmann, On the autoconvolution equation and total variation constraints, Z. Angew. Math. Mech 79 (1999) 149-159.

[14] R. Gorenflo, B. Hofmann, On autoconvolution and regularization, Inverse Problems 10 (1994) 353-373.

[15] J. Janno, On a regularization method for the autoconvolution equation, Z. Angew. Math. Mech $\mathbf{7 7}$ (1997) 393-394.

[16] J. Janno, Lavrent'ev regularization of ill-posed problems containing nonlinear near-tomonotone operators with application to autoconvolution equation, Inverse Problems 16 (2000) 333-348.

[17] P. K. Lamm, Approximation of ill-posed Volterra problems via predictor-corrector regularization methods, SIAM J. Appl. Math. 195 (1995) 469-494.

[18] P. K. Lamm, Future-sequential regularization methods for ill-posed Volterra equations: applications to the inverse heat conduction problem, J. Math. Anal. Appl. 56 (1996) 524-541.

[19] P. K. Lamm, Regularized inversion of finitely smoothing Volterra operators: predictor-corrector regularization methods, Inverse Problems 13 (1997) 375-402.

[20] P. K. Lamm, Variable-smoothing regularization methods for inverse problems, Theory and Practice of Control and Systems ed A. Conte and A. M. Perdon (Singapore: World Scientific) (1999).

[21] P. K. Lamm, Variable-smoothing local regularization methods for first-kind integral equations, Inverse Problems 19 (2003) 195-216.

[22] P. K. Lamm, Full convergence of sequential local regularization methods for Volterra inverse problems, Inverse Problems 21 (2005) 785-803.

[23] P. K. Lamm and Z. Dai, On local regularization methods for linear Volterra problems and nonlinear equations of Hammerstein type, Inverse Problems, 21 (2005) 1773-1790.

[24] P. K. Lamm, A survey of regularization methods for first-kind Volterra equations, Surveys on Solution Methods for Inverse Problems, eds D. Colton, H. W. Engl, A. Louis, J. R. McLaughlin, W. Rundell (Vienna, New York: Springer) (2000) 53-82.

[25] P. K. Lamm and L. Eldén, Numerical solution of first-kind Volterra equations by sequential Tikhonov regularization, SIAM J. Numer. Anal. 34 (1997) 1432-1450.

[26] P. K. Lamm and X. Luo, Local regularization methods for nonlinear Hammerstein equations. Preprint (2006).

[27] P. K. Lamm and T. Scofield, Sequential predictor-corrector methods for the variable regularization of Volterra inverse problems, Inverse Problems 16 (2000) 373-399.

[28] P. K. Lamm and T. Scofield, Local regularization methods for the stabilization for ill-posed Volterra problems, Numer. Funct. Analy. Opt. 23 (2001) 913-940.

[29] R. Miller, Nonlinear Volterra integral equations, W. A. Benjamin, Inc (1971).

[30] R. Ramlau, Morozov's discrepancy principle for Tikhonov regularization of nonlinear operators, Numer. Funct. Analy. Opt. 23 (2002) (1\&2) 147-172.

[31] W. Ring and J. Prix, Sequential predictor-corrector regularization methods and their limitations, Inverse Problem 16 (2000) 619-634.

[32] O. Scherzer, The use of Morozov's discrepancy principle for Tikhkonov regularization for solving nonlinear ill-posed problems, Computing 51 (1993) 45-60.

[33] H. Tanabe, Equations of evolution, London:Pitman, (1979). 